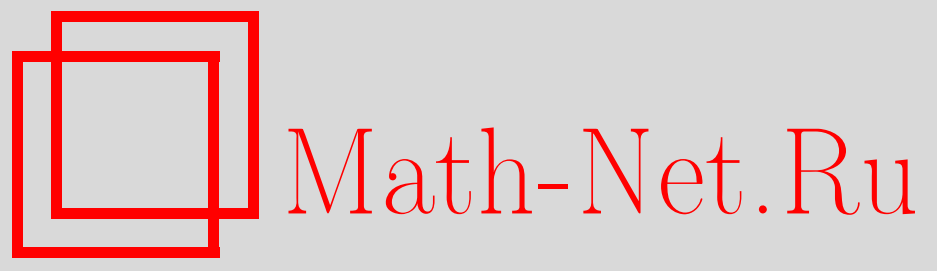

Э. Э. Гасанов, И. В. Кузнецова, О функциональной сложности двумерной задачи интервального поиска, Дискрет. матем., 2002, том 14, выпуск 1, 114-141

DOI: https://doi.org/10.4213/dm226

Использование Общероссийского математического портала Math-Net.Ru подразумевает, что вы прочитали и согласны с пользовательским соглашением http://www . mathnet.ru/rus/agreement

Параметры загрузки:

IP : 3.89.185.249

26 апреля 2023 г., 13:07:54

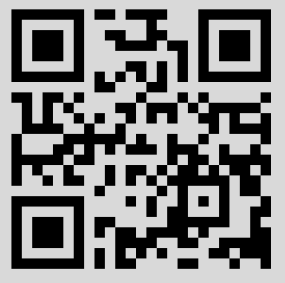




\title{
О функциональной сложности двумерной задачи интервального поиска
}

\author{
(C) 2002 г. Э. Э. Гасанов, И. В. Кузнецова
}

\begin{abstract}
Предлагается модификация алгоритма Бентли-Маурера, решающего двумерную задачу интервального поиска. Эта модификация позволяет снизить исходное логарифмическое среднее время поиска до константного при сохранении логарифмического времени поиска в худшем случае. Этот алгоритм зависит от параметра, при вариации которого объем памяти, необходимый алгоритму, изменяется от $O\left(k^{3}\right)$ до $O(k \log k)$, при этом среднее время поиска (без учета времени на перечисление ответа) изменяется от $O(1)$ до $O(\log k)$. В частности, для любого $\varepsilon>0$ при объеме памяти $O\left(k^{1+\varepsilon}\right)$ достигается среднее время поиска $O(1)$. На основе этих результатов получены верхние оценки функциональной сложности двумерной задачи интервального поиска.

Работа выполнена при поддержке Российского фонда фундаментальных исследований, гранты 98-01-00130, 01-01-00748.
\end{abstract}

\section{1. Введение}

Данная работа посвящена исследованию сложности алгоритмов решения двумерной задачи интервального поиска, которая состоит в поиске в конечном подмножестве евклидовой плоскости всех тех точек, которые попадают в прямоугольник-запрос.

Типичная задача интервального поиска для двух измерений заключается в выявлении в базе данных, содержащей записи о служащих некоторой компании, всех служащих, чьи возраст и жалованье находятся в заданньх интервалах. Это пример взят из [1]. Другой пример можно найти в [2], где рассматривается база данных городов США с координатами в виде ширсты и долготы. $\mathrm{K}$ такой базе естественен вопрос о перечислении всех городов, попадающих в некоторой прямоугольник-запрос. Подобные задачи возникают также в статистике [3] и автоматизации проектирования [4].

Исследованию задачи интервального поиска (в другом переводе с английского регионального поиска) посвящено большое количество работ $[1,5,6,7,8,9,10,11,12$, $13,14,15,16,17,18,19,20,21,22]$. Приведем результаты некоторых из них. Алгоритм решения двумерной задачи интервального поиска путем сведения к решению четырех двумерных задач о доминировании можно найти в $[1,6]$. В [7] предложен метод многомерного двоичного дерева ( $k$ - $D$-дерева), исходя из того, что бинарное дерево для одномерной задачи дает хороший результат. Этот метод имеет линейные затраты по памяти, но в [17] показано, что в худшем случае этим алгоритмом двумерная

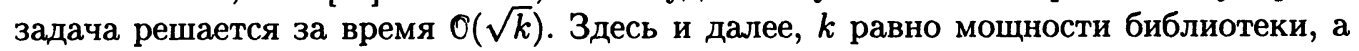


оценки приводятся без времени перечисления ответа. В [8] предложен метод прямого доступа, который решает задачу за время $\odot(\log k)$, но требует затрат по памяти порядка $k^{3}$. Чтобы уменьшить требуемую память, в [10] предложен многоэтапный метод прямого доступа. Он позволяет снижать порядок требуемой памяти, но при этом возрастает константа при логарифме в оценке времени. С помощью метода дерева интервалов (или дерева регионов) в [11] получена оценка времени поиска $O\left(\log ^{2} k\right)$ при затратах памяти $\odot(k \log k)$. В [22] и [19] независимо предложена модификация дерева интервалов, которая позволила снизить время поиска до $\mathcal{O}(\log k)$ при тех же затратах памяти. В [14] затраты памяти снижены до $O(k \log k / \log \log k)$, но при этом возросла константа при логарифме в оценке времени. Во всех этих работах оценивается время поиска в худшем случае. В [13] описан метод хеширования, который использован вместо поиска по древовидным структурам в задаче интервального поиска. Применение этого метода позволило получить довольно быстрые в среднем решения задачи интервального поиска при условии, что область запросов находится в заранее определенных границах.

При оценке алгоритмов поиска обычно используют три основные характеристики: объем памяти, требуемый алгоритмом, время поиска в худшем случае и среднее время поиска. Естественно, хотелось бы находить алгоритмы, хорошие в соответствии с той или иной характеристикой, или, возможно, в соответствии с комбинацией характеристик. Выбор критерия оптимизации является одним из основных моментов в теории синтеза управляющих систем (в частности, при синтезе оптимальных алгоритмов поиска). Условимся обозначать через $Q$ объем требуемой памяти, а через $T$ время вычислений. В литературе в первую очередь встречаются такие критерии оптимизации: $Q, T, Q \cdot T, Q \cdot T^{2}$. Выбор конкретного критерия оптимизации осуществляется на практике с учетом специфики разрабатываемой вычислительной системы и особенностей ее использования, и многообразие таких критериев подсказывает необходимость проведения исследований глобальных зависимостей $Q(T)$ или $T(Q)$. Такая мера сложности носит название функциональной и была предложена А. С. Подколзиным [24].

Понятно, что любой алгоритм, который имеет оценки объема памяти и времени работы, дает верхнюю оценку функциональной сложности. В данной работе предлагается алгоритм решения двумерной задачи интервального поиска, зависящий от некоторого параметра. При вариации этого параметра получаются разные соотношения объема памяти и среднего времени поиска, что позволяет оценить функциональную сложность в некоторой последовательности точек. Данный алгоритм является модификацией алгоритма Бентли-Маурера, изложенного в [10], но в отличие от исходного алгоритма предлагаемый алгоритм при сохранении порядка оценок объема памяти и времени в худшем случае имеет константные оценки времени в среднем.

Результаты данной работы были анонсированы в [25, 23].

Авторы выражают благодарность В. Б. Кудрявцеву и А. С. Подколзину за поддержку в работе.

\section{2. Основные понятия и формулировка результата}

Мы будем использовать терминологию и обозначения из работы [26].

Пусть $X$ - множество запросов с заданным на нем вероятностным пространством $\langle X, \sigma, \mathbf{P}\rangle$, где $\sigma$ - алгебра подмножеств множества $X, \mathbf{P}$ - вероятностная мера 
на $\sigma, Y$ - множество записей (объектов поиска), $\rho$ - бинарное отношение на $X \times Y$, называемое отношением поиска.

Пятерку $S=\langle X, Y, \rho, \sigma, \mathbf{P}\rangle$ будем называть типом. Тройку $I=\langle X, V, \rho\rangle$, где $V$ - некоторое конечное подмножество множества $Y$, в дальнейшем называемое библиотекой, будем называть задачей информационного поиска (ЗИП) типа $S$, и будем считать, что ЗИП $I=\langle X, V, \rho\rangle$ содержательно состоит в перечислении для произвольно взятого запроса $x \in X$ всех тех и только тех записей $y \in V$ таких, что $x \rho y$.

Пусть $f$ - одноместный предикат, определенный на $X$, то есть $f: X \rightarrow\{0,1\}$. Множество $N_{f}=\{x \in X: f(x)=1\}$ назовем характеристическим множеством предиката $f$.

Множество $O(y, \rho)=\{x \in X: x \rho y\}$ назовем тенью записи $y \in Y$.

Функцию $\chi_{y, \rho}: X \rightarrow\{0,1\}$ такую, что $N_{\chi_{y, \rho}}=O(y, \rho)$ назовем характеристической функцией записи $y$.

Пусть $F$ - множество символов одноместных предикатов, определенных на множестве $X, G$ - множество символов одноместных переключателей, определенных на множестве $X$. Под переключателем будем понимать функцию, областью значений которой является конечное подмножество натурального ряда. Пару $\mathscr{F}=\langle F, G\rangle$ назовем базовым множеством.

Понятие информационного графа (ИГ) над базовым множеством $\mathscr{F}=\langle F, G\rangle$ определяется следующим образом. Берется конечная многополюсная ориентированная сеть. В ней выбирается некоторый полюс, который называется корнем. Остальные полюсы называются листьями и им приписываются записи из $Y$, причем разным листьям могут быть приписаны одинаковые записи. Некоторые вершины сети (в том числе это могут быть и полюсы) называются переключательными и им приписываются переключатели из $G$. Ребра, исходящие из каждой из переключательных вершин, нумеруются подряд, начиная с 1 , и называются переключательными ребрами. Ребра, не являющиеся переключательными, называются предикатными и им приписываются предикаты из множества $F$. Таким образом нагруженную многополюсную ориентированную сеть называем ИГ над базовым множеством $\mathscr{F}=\langle F, G\rangle$.

Функционирование ИГ определяется следующим образом. Скажем, что предикатное ребро проводит запрос $x \in X$, если предикат, приписанный этому ребру, принимает значение 1 на запросе $x$. Переключательное ребро, которому приписан номер $n$, проводит запрос $x \in X$, если переключатель, приписанный началу этого ребра, принимает значение $n$ на запросе $x$. Ориентированная цепочка ребер проводит запрос $x \in X$, если каждое ребро цепочки проводит запрос $x$. Запрос $x \in X$ проходит в вершину $\beta$ ИГ, если существует ориентированная цепочка, ведущая из корня в вершину $\beta$, которая проводит запрос $x$. Запись $y$, приписанная листу $\alpha$, попадает в ответ ИГ на запрос $x \in X$, если запрос $x$ проходит в лист $\alpha$. Ответом ИГ $U$ на запрос $x$ назовем множество записей, попавших в ответ ИГ на запрос $x$, и обозначим его $\mathscr{F}_{U}(x)$. Эту функцию $\mathscr{F}_{U}(x)$ будем считать результатом функционирования ИГ U.

Пусть дана ЗИП $I=\langle X, V, \rho\rangle$. Скажем, что ИГ $U$ разрешает ЗИП $I=\langle X, V, \rho\rangle$, если $\mathscr{S}_{U}(x)=\{y \in V: x \rho y\}$. ИГ $U$, разрешающий ЗИП $I$, будем также называть допустимым для $I$.

Введем понятие сложности ИГ. Пусть $\beta$ - некоторая вершина ИГ. Предикат, определенный на множестве запросов, который принимает значение 1 на запросе $x$, если запрос проходит в вершину $\beta$, и 0 в противном случае, назовем функцией 
фильтра вершины $\beta$ и обозначим $\varphi_{\beta}(x)$.

Сложностью ИГ $U$ на запросе $x \in X$ назовем число

$$
T(U, x)=\sum_{\beta \in \mathscr{P}} \varphi_{\beta}(x)+\sum_{\beta \in \mathscr{R} \backslash \mathscr{P}} \psi_{\beta} \varphi_{\beta}(x)
$$

где $\mathscr{R}$ - множество вершин ИГ $U, \mathscr{P}$ - множество переключательных вершин ИГ $U, \psi_{\beta}-$ количество ребер, исходящих из вершины $\beta$.

Скажем, что базовое множество F्F измеримо, если каждая функция из FF измерима (относительно алгебры $\sigma$ ). Далее всюду будем предполагать, что базовое множество измеримо. В этом случае для любого ИГ $U$ над $\mathscr{F}$ функция $T(U, x)$ как функция от $x$ измерима.

Сложностью ИГ $U$ назовем математическое ожидание величины $T(U, x)$, то есть число

$$
T(U)=\mathrm{M}_{x} T(U, x) .
$$

Объемом $Q(U)$ ИГ $U$ назовем число ребер в графе $U$. Сложностью задачи $I$ при базовом множестве F्F назовем число

$$
T(I, \mathscr{F})=\inf \{T(U): U \in \mathcal{U}(I, \mathscr{F})\}
$$

где $U(I, \mathscr{F})$ - множество всех ИГ над базовым множеством $\mathscr{F}$, разрешающих ЗИП $I$.

Дадим описание типа задач поиска, который соответствует двумерной задаче интервального поиска.

Пусть $Y_{\text {int } 2}=[0,1)^{2}-$ множество записей и

$$
X_{\text {int } 2}=\left\{\tilde{x}=\left(u_{1}, v_{1}, u_{2}, v_{2}\right): 0 \leqslant u_{i} \leqslant v_{i}<1, i=1,2\right\}
$$

- множество запросов. Пусть на множестве $X_{\text {int } 2}$ задано вероятностное пространство $\left\langle X_{\text {int } 2}, \sigma, \mathbf{P}\right\rangle$, где $\mathbf{P}$ задается функцией плотности вероятности $p(\tilde{x})$.

Отношение поиска $\rho_{\text {int } 2}$ определено на $X_{\text {int } 2} \times Y_{\text {int } 2}$ и задается соотношением

$$
\left(u_{1}, v_{1}, u_{2}, v_{2}\right) \rho_{\text {int } 2}\left(y_{1}, y_{2}\right) \Longleftrightarrow u_{i} \leqslant y_{i} \leqslant v_{i}, \quad i=1,2 \text {. }
$$

Тогда тип

$$
S_{\text {int 2 }}=\left\langle X_{\text {int 2 }}, Y_{\text {int 2 }}, \rho_{\text {int 2 }}, \sigma, \mathbf{P}\right\rangle
$$

назовем типом двумерного интервального поиска.

Пусть

$$
\begin{aligned}
& G_{1}=\left\{g_{i, a, b}^{1, m}(\tilde{x})=\left[\left(u_{i}-a\right) m /(b-a)\right]+1, i=1,2, m \in \mathbf{N}, 0 \leqslant a<b<1\right\}, \\
& G_{2}=\left\{g_{a, b}^{2, m}(\tilde{x})=\left[\left(v_{1}-a\right) m /(b-a)\right]+1, m \in \mathbf{N}, 0 \leqslant a<b<1\right\}, \\
& G_{3}=\left\{g_{i, a}^{3}(\tilde{x})=\left\{\begin{array}{ll}
1, & \text { если } u_{i} \leqslant a, \\
2, & \text { если } u_{i}>a,
\end{array} \quad i=1,2, a \in[0,1)\right\},\right. \\
& G_{4}=\left\{g_{a}^{4}(\tilde{x})=\left\{\begin{array}{ll}
1, & \text { если } v_{1}<a, \\
2, & \text { если } v_{1} \geqslant a,
\end{array} \quad a \in[0,1)\right\},\right. \\
& G_{5}=\left\{g_{m}^{5}(\tilde{x})=\left\{\begin{array}{ll}
1, & \text { если } 0 \leqslant v_{2}-u_{2}<1 / m, \\
2 & \text { в противном случае, }
\end{array} \quad m \in \mathbf{N}\right\},\right.
\end{aligned}
$$




$$
\begin{aligned}
& G_{6}=\left\{g_{a, b}^{6}(\tilde{x})=\left\{\begin{array}{ll}
1, & \text { если } a<u_{1} \leqslant v_{1}<b, \\
2 & \text { в противном случае, }
\end{array} \quad 0 \leqslant a<b<1\right\},\right. \\
& F_{1}=\left\{f_{a}(\tilde{x})=\left\{\begin{array}{ll}
1, & \text { если } u_{2} \leqslant a \leqslant v_{2}, \\
0 & \text { в противном случае, }
\end{array} \quad a \in[0,1)\right\} .\right.
\end{aligned}
$$

Введем базовое множество

$$
\mathscr{F}=\left\langle F_{1}, G_{1} \cup G_{2} \cup G_{3} \cup G_{4} \cup G_{5} \cup G_{6}\right\rangle .
$$

Для удобства будем считать, что функция плотности вероятности $p(\tilde{x})=$ $p\left(u_{1}, v_{1}, u_{2}, v_{2}\right)$, определяющая вероятностную меру $\mathbf{P}$ вероятностного пространства над $X_{\text {int 2 }}$, задана на $[0,1)^{2}$, но вне $X_{\text {int } 2}$ она принимает значение 0.

Введем обозначения

$$
\begin{gathered}
X_{\mathrm{int}}=\{(u, v) \mid 0 \leqslant u \leqslant v<1\} \\
p_{1}\left(u_{1}, v_{1}\right)=\int_{X_{\mathrm{int}}} p(\tilde{x}) d \tilde{z}_{2}, \quad p_{2}\left(u_{1}, v_{1}, u_{2}, v_{2}\right)=p(\tilde{x})
\end{gathered}
$$

где $\tilde{z}_{i}=\left(u_{i}, v_{i}\right), i=1,2$.

Пусть $b, d, e, f \in[0,1)$ и $b<d, e<f, b \leqslant e$ и

$$
\begin{aligned}
p^{1}\left(u_{1}, v_{1}\right) & =p_{1}\left(u_{1}, v_{1}\right), \\
p_{b, d, e, f}^{2}\left(u_{2}, v_{2}\right) & =\int_{b}^{d} d u_{1} \int_{e}^{f} p_{2}\left(u_{1}, v_{1}, u_{2}, v_{2}\right) d v_{1} / \int_{b}^{d} d u_{1} \int_{e}^{f} p_{1}\left(u_{1}, v_{1}\right) d v_{1}, \\
p^{1, b, d, e, f}\left(u_{1}\right) & =\int_{e}^{f} p^{1}\left(u_{1}, v_{1}\right) d v_{1} / \int_{b}^{d} d u_{1} \int_{e}^{f} p^{1}\left(u_{1}, v_{1}\right) d v_{1} \\
p^{2, b, d, e, f}\left(v_{1}\right) & =\int_{b}^{d} p^{1}\left(u_{1}, v_{1}\right) d u_{1} / \int_{b}^{d} d u_{1} \int_{e}^{f} p^{1}\left(u_{1}, v_{1}\right) d v_{1} .
\end{aligned}
$$

Пусть $c$ - некоторая вещественная константа. Скажем, что функция плотности вероятности $p(\tilde{x})=p\left(u_{1}, v_{1}, u_{2}, v_{2}\right)$ обладает свойством $c$-ограниченности, если для любых $b, d, e, f \in[0,1)$ таких, что $b<d, e<f, b \leqslant e$ выполняются условия

$$
\begin{gathered}
(d-b)(f-e) p_{b, d, e, f}^{2}\left(u_{2}, v_{2}\right) \leqslant c \\
(d-b)(f-e) p^{1, b, d, e, f}\left(u_{1}\right) \leqslant c \\
(d-b)(f-e) p^{2, b, d, e, f}\left(v_{1}\right) \leqslant c .
\end{gathered}
$$

Tеорема 1. Пусть $I=\left\langle X_{\text {int } 2}, V, \rho_{\text {int } 2}\right\rangle$-двумерная задача интервального поиска, где $|V|=k$. Пусть $\mathscr{F}$ - базовое множество, определяемое соотношениями (1)-(8). $\Pi y c m s$

$$
R(I)=\sum_{\tilde{y} \in V} \mathbf{P}\left(O\left(\tilde{y}, \rho_{\text {int } 2}\right)\right) .
$$

Тогда если для нехоторой константы с функция плотности вероятности $p(\tilde{x})$, определяющая вероятностную меру $\mathbf{P}$, обладает свойством с-ограниченности, то для любого натурального $M$ тахого, что $1 \leqslant M \leqslant 2 \ln k$,

$$
R(I) \leqslant T\left(I, \mathscr{F},(2 / 3) M k^{1+2 / M}+\mathcal{O}\left(k^{1+1 / M}(M+(\ln k) / M)\right)\right) \leqslant R(I)+16 M-6,
$$


причем для ИГ $U^{*}$, на котором достигается верхняя оченка, для любого $\tilde{x} \in X_{\mathrm{int} 2}$ справедлива оченка

$$
T\left(U^{*}, \tilde{x}\right)<10 M+4 \log _{2} k+\left|\left\{\tilde{y} \in V: \tilde{x} \rho_{\text {int } 2} \tilde{y}\right\}\right|
$$

При вариации параметра $M$ мы получаем серию оценок функциональной сложности задачи в различных точках. Так, если $M=2$, то при объеме памяти $Q=4 k^{2} / 3$ среднее время поиска (без времени перечисления ответа) равно $T=26$. Для любой константы $\varepsilon>0$ можно выбрать параметр $M=] 2 / \varepsilon[$ так, что при объеме памяти $Q=O\left(k^{1+\varepsilon}\right)$ среднее время равно $\left.T=16 M-6=16\right] 2 / \varepsilon[-6=$ const. Ограничение сверху на параметр $M$, приведенное в теореме, объясняется тем, что функция $Q_{1}(M)=M k^{1+2 / M}$ имеет точку минимума $M=2 \ln k$, функция $Q_{2}(M)=M k^{1+1 / M}$ имеет точку минимума $M=\ln k$, функция $Q_{3}(M)=((\ln k) / M) k^{1+1 / M}$ является убывающей по $M$, причем $Q_{3}(\ln k)=e k$. Поэтому после точки $M=2 \ln k$ объем $Q(M)$ данного метода заведомо будет возрастать при одновременном возрастании среднего времени поиска $T(M)$. Поэтому при минимальном по порядку для предлагаемого метода объеме $Q=\mathcal{O}(k \log k)$ среднее время есть $T=\mathcal{O}(\log k)$, что соответствует по порядку методу Уилларда [22] и Люкера [19], но в силу сложности своих конструкций предлагаемый метод в данном случае является менее предпочтительным, чем метод Уилларда-Люкера.

Нижняя оценка в теореме следует из теоремы 4 в [27]. Смысл нижней оценки в том, что время поиска не может быть меньше времени, необходимого на перечисление ответа.

Ниже будет приведено доказательство справедливости указанной выше верхней оценки.

\section{3. Неформальное описание алгоритма}

Пусть абсциссы точек библиотеки

$$
V=\left\{\tilde{y}_{1}, \ldots, \tilde{y}_{k}\right\}, \quad \tilde{y}_{i}=\left(y_{i}^{1}, y_{i}^{2}\right), \quad i=1, \ldots, k,
$$

упорядочены по возрастанию:

$$
y_{1}^{1} \leqslant y_{2}^{1} \leqslant \ldots \leqslant y_{k}^{1}
$$

Для упрощения изложения будем считать, что все точки $y_{i}^{1}$ различны, поскольку при их совпадении сложность получаемых ИГ только уменьшается.

Пусть $M \in \mathbf{N}, M \leqslant k$ и $\alpha_{1}, \ldots, \alpha_{M}, 0<\alpha_{i} \leqslant 1, i=1, \ldots, M$ - некоторые рациональные числа такие, что

$$
\sum_{i=1}^{M} \alpha_{i}=1
$$

Для любого $q \in\{1, \ldots, M\}$ положим $l_{0}^{q}=0, l_{k^{\alpha_{1}+\ldots+\alpha_{q}+1}}^{q}=1$, причем здесь и везде далее, если некоторое действительное число используется как целое, то подразумевается, что используется целая часть этого числа. Множество

$$
\mathcal{N}^{q}=\left\{l_{j}^{q} \mid l_{j}^{q}=y_{(j-1) k^{1-\left(\alpha_{1}+\ldots+\alpha_{q}\right)}+1}^{1}, j=1, \ldots, k^{\alpha_{1}+\ldots+\alpha_{q}}\right\} .
$$


Рис. 1. Случай $s \leqslant t$

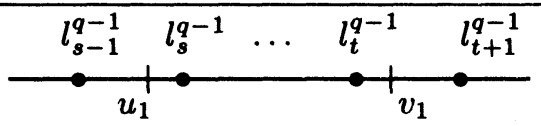

назовем $q$-й сеткой, а интервалы вида

$$
\left[l_{j}^{q}, l_{j+1}^{q}\right), \quad j=1, \ldots, k^{\alpha_{1}+\ldots+\alpha_{q}},
$$

назовем интервалами $q$-й сетки. Точки $q$-й сетки делят каждый интервал $(q-1)$-й сетки на $k^{\alpha_{q}}$ частей. В каждом интервале $q$-й сетки содержится $k^{1-\left(\alpha_{1}+\ldots+\alpha_{q}\right)}$ точек из множества $\left\{y_{1}^{1}, \ldots, y_{k}^{1}\right\}, l_{j}^{q-1}=l_{(j-1) k^{\alpha_{4}+1}}^{q}$. Очевидно, самая последняя сетка будет иметь вид

$$
\mathcal{N}^{M}=\left\{l_{j}^{M}=y_{j}^{1}, \quad j=1, \ldots, k\right\} .
$$

Для $M>1$ и $q \in\{2, \ldots, M\}$ положим

$$
\mathcal{N}_{s}^{q}=\left\{l_{j}^{q} \in \mathcal{N}^{q} \mid l_{j}^{q} \in\left(l_{s}^{q-1}, l_{s+1}^{q-1}\right)\right\}, \quad s=1, \ldots, k^{\alpha_{1}+\ldots+\alpha_{q-1}} .
$$

Под одномерным интервальным поиском понимается задача типа

$$
S_{\mathrm{int}}=\left\langle X_{\mathrm{int}},[0,1), \rho_{\mathrm{int}}\right\rangle
$$

где $\rho_{\text {int }}$ определяется соотношением

$$
(u, v) \rho_{\mathrm{int}} y \Longleftrightarrow u \leqslant y \leqslant v .
$$

Поиск по запросу $\tilde{x}=\left(u_{1}, v_{1}, u_{2}, v_{2}\right)$ предлагаемым алгоритмом проводится в $M$ этапов.

\section{1-й этап.}

(1) Среди точек множества $\mathcal{N}^{1} \cup\{1\}$ ищется точка, ближайшая справа к $u_{1}$. Пусть это $l_{p}^{1}, p \in\left\{1, \ldots, k^{\alpha_{1}}+1\right\}$.

(2) Среди точек множества $\left\{l_{j}^{1} \mid j=p-1, \ldots, k^{\alpha_{1}}\right\}$ ищется точка, ближайшая слева к $v_{1}$. Пусть это $l_{r}^{1}, r \in\left\{p-1, \ldots, k^{\alpha_{1}}\right\}$.

(3) Если $p<r$, то для упорядоченных ординат $y_{j}^{2}$ тех точек библиотеки $V$, абсциссы которых удовлетворяют условию $l_{p}^{1} \leqslant y_{j}^{1}<l_{r}^{1}$, мы решаем одномерную задачу интервального поиска, где в качестве запроса берется отрезок $\tilde{x}=\left[u_{2}, v_{2}\right]$.

(4) Если $M=1$ и $l_{r}^{1}=y_{i}^{1}$ для некоторого $i \in\{1, \ldots, k\}$, то проверяем принадлежность ординаты $y_{i}^{2}$ отрезку $\left[u_{2}, v_{2}\right]$ и в зависимости от результата проверки включаем или не включаем точку $\tilde{y}_{i}=\left(y_{i}^{1}, y_{i}^{2}\right)$ в ответ задачи.

(5) Если $M>1$, то переходим ко второму этапу. 
Рис. 2. Случай $t=s-1$

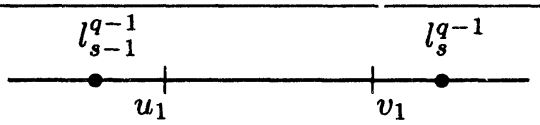

$q$-й этап, $2 \leqslant q \leqslant M$. Пусть $l_{s}^{q-1}-$ точка, ближайшая справа к $u_{1}$ на $(q-1)$-й сетке, $l_{t}^{q-1}$ - точка, ближайшая слева к $v_{1}$ на $(q-1)$-й сетке. Эти точки являются левыми концами $s$-го и $t$-го интервалов $(q-1)$-й сетки соответственно.

(1) Если $s \leqslant t$ (см. рис. 1), то

(1.1) среди точек множества $\mathcal{N}_{s-1}^{q}$ ищем точку, ближайшую справа к $u_{1}$, обозначим найденную точку $\xi^{\prime}$;

(1.2) среди точек множества $\mathcal{N}_{t}^{q}$ ищем точку, ближайшую слева к $v_{1}$, обозначим найденную точку $\xi^{\prime \prime}$;

(1.3) для упорядоченных ординат $y_{j}^{2}$ тех точек библиотеки $V$, абсциссы которых $y_{j}^{1}$ удовлетворяют условию $\xi^{\prime} \leqslant y_{j}^{1}<l_{s}^{q-1}$, решаем одномерную задачу интервального поиска с запросом $\tilde{x}^{\prime}=\left[u_{2}, v_{2}\right]$;

(1.4) для упорядоченных ординат $y_{j}^{2}$ тех точек библиотеки $V$, абсциссы которых $y_{j}^{1}$ удовлетворяют условию $l_{t}^{q-1} \leqslant y_{j}^{1}<\xi^{\prime \prime}$, решаем одномерную задачу интервального поиска с запросом $\tilde{x}^{\prime}=\left[u_{2}, v_{2}\right]$.

(2) Если $t=s-1$ (см. рис. 2), то

(2.1) среди точек множества $\mathcal{N}_{s-1}^{q}$ ищем точку, ближайшую справа к $u_{1}$, обозначим найденную точку $\xi^{\prime}$;

(2.2) пусть $\xi$ - точка, предшествующая точке $\xi^{\prime}$ на $q$-й сетке, среди точек множества $\mathcal{N}_{s-1}^{q}$, не меньших $\xi$, ищем точку, ближайшую слева к $v_{1}$, обозначим ee $\xi^{\prime \prime}$

(2.3) если $\xi^{\prime}<\xi^{\prime \prime}$, то для упорядоченных ординат $y_{j}^{2}$ тех точек библиотеки $V$, абсциссы которых $y_{j}^{1}$ удовлетворяют условию $\xi^{\prime} \leqslant y_{j}^{1}<\xi^{\prime \prime}$, решаем одномерную задачу интервального поиска, где в качестве запроса берется отрезок $\tilde{x}^{\prime}=\left[u_{2}, v_{2}\right]$.

(3) Если $q=M$ и $\xi^{\prime \prime}=y_{i}^{1}$ для некоторого $i \in\{1, \ldots, k\}$, то проверяем принадлежность ординаты $y_{i}^{2}$ отрезку $\left[u_{2}, v_{2}\right]$ и в зависимости от результата проверки включаем или не включаем точку $\tilde{y}_{i}=\left(y_{i}^{1}, y_{i}^{2}\right)$ в ответ задачи.

(4) Если $q<M$, то осуществляем переход к следующему этапу.

Для решения одномерной задачи интервального поиска и задачи о нахождении точки, ближайшей к данной (задача о близости) воспользуемся алгоритмами, приведенными в [27]. Дадим их неформальное описание.

\section{1. Одномерный интервальный поиск}

Пусть $V=\left\{z_{1}, \ldots, z_{l}\right\} \subset[0,1)-$ упорядоченное по возрастанию множество, в котором мы должны производить поиск (библиотека), $X_{\text {int }}$ - множество запросов, $m \in \mathbf{N}$ - некоторый параметр, значение которого будет установлено позже. Построим для библиотеки $V$ множество номеров $S=\left\{s_{1}, \ldots, s_{m-1}\right\}$, где $s_{i}$ - номер записи 
из $V$ такой, что $z_{s_{i}}-$ ближайшая слева запись к точке $i / m, i=1, \ldots, m-1$. Если такой записи нет, то $s_{i}=0$. Отметим, что это множество строится только один раз.

Теперь поиск по произвольному запросу $\tilde{x}=(u, v)$ производится следующим образом.

Сначала вычисляется длина запроса $\tilde{x}$.

Если она меньше, чем $1 / m$, то в множестве $V$ дихотомическим поиском находится ближайшая справа к точке $u$ запись. Далее, начиная с этой записи, просматриваются слева направо все записи из $V$ и сравниваются с правым концом запроса - точкой $v$ до тех пор, пока очередная запись не станет больше $v$.

Если $v-u \geqslant 1 / m$, то вычисляем номер $j=[n \cdot m]+1$. Понятно, что точка $j / m$ попадает в интервал $[u, v]$. Теперь, начиная с записи с номером $s_{j}$, просматриваем справа налево записи из $V$ и сравниваем с левым концом запроса - точкой $u$. Как только очередная запись окажется меньше $u$, начиная с записи с номером $s_{j}+1$, просматриваем слева направо записи из $V$ и сравниваем с правым концом запроса -точкой $v$ до тех пор, пока очередная запись не станет больше $v$.

\section{2. Поиск точки, ближайшей к данной}

Пусть $V=\left\{z_{1}, \ldots, z_{l}\right\} \subset[a, b)$ - упорядоченное по возрастанию множество, в котором мы должны производить поиск (библиотека), $X=[a, b)$ - множество запросов, $m \in \mathbf{N}$ - некоторый параметр, значение которого будет установлено позже.

Разобьем множество $X=[a, b)$ на $m$ равных частей $X_{1}, \ldots, X_{m}$, где

$$
X_{i}=[a+(i-1)(b-a) / m, a+i(b-a) / m), \quad i=1, \ldots, m .
$$

Пусть $V_{i}=X_{i} \cap V, i=1, \ldots, m$.

Теперь для произвольной точки-запроса $x$ поиск точки из $V$, ближайшей справа (слева) к $x$, будем проводить следующим образом.

Определим ту часть отрезка, которой принадлежит запрос $x$. Ее номер равен $i=$ $[(x-a) m /(b-a)]+1$, после чего в множестве $V_{i}$ осуществим обычный дихотомический поиск.

Очевидно, что при $M=1$ описанный выше многоэтапный алгоритм совпадает с алгоритмом решения двумерной задачи интервального поиска из [27].

\section{4. Построение информационного графа}

Прежде, чем приступить к построению ИГ над базовым множеством $\mathscr{F}$, решающего двумерную задачу интервального поиска описанным выше методом, введем вспомогательные блоки, из которых и будем впоследствии строить ИГ.

Пусть $[b, d] \subseteq[0,1)-$ произвольный интервал.

Пусть $Z=\left\{z_{1}, \ldots, z_{l}\right\}$ - упорядоченное множество действительных чисел из интервала $[b, d]$. Через $U_{Z, b, d}^{1}$ обозначим ИГ с одной входной вершиной (корнем) и $l+1$ выходными вершинами (листьями, которые занумерованы слева направо числами $1, \ldots, l+1)$ такой, что функция проводимости между корнем и $i$-м, $i=1, \ldots, l+1$, листом имеет вид

$$
f_{i}\left(u_{1}, v_{1}, u_{2}, v_{2}\right)= \begin{cases}1, & \text { если } z_{i-1}<u_{1} \leqslant z_{i} \\ 0 & \text { в противном случае }\end{cases}
$$


где $z_{0}=b, z_{l+1}=d$.

Если считать, что $i$-му листу, $i=1, \ldots, l+1$, соответствует точка $z_{i}$, то $U_{Z, b, d}^{1}$ позволяет находить точку из множества $\left\{z_{1}, \ldots, z_{l}, z_{l+1}=d\right\}$, ближайшую справа к $u_{1}$.

ИГ $U_{Z, b, d}^{1}$ строится по методу, описанному в параграфе 2.3 в [27] с помощью функций из $G_{1} \cup G_{3}$. Неформальное описание алгоритма соответствующего ИГ $U_{Z, b, d}^{1}$ (то есть решающего задачу поиска ближайшей точки) приведено в предыдущем разделе.

Вероятностная мера на множестве запросов $u_{1} \in[b, d]$ при условии, что $b \leqslant e \leqslant$ $v_{1} \leqslant f<1$, в рассмотренной задаче о близости определяется функцией плотности вероятности, задаваемой соотношением (10). Поскольку справедливо (13), мы находимся в условиях теоремы 7 из [27]. Следовательно, если выбрать в качестве параметра алгоритма (см. предыдущий раздел) $m=] c l[$, то

$$
\left.Q\left(U_{Z, b, d}^{1}\right)=(2+c) l+1, \quad T\left(U_{Z, b, d}^{1}\right) \leqslant 2, \quad T\left(U_{Z, b, d}^{1}, x\right) \leqslant 1+\right] \log _{2}(l+1)[
$$

для любого $x \in[b, d]$.

Пусть $Z=\left\{z_{1}, \ldots, z_{l}\right\}-$ упорядоченное множество действительных чисел из интервала $[e, f]$. Через $U_{Z, e, f}^{2}$ обозначим ИГ с одной входной вершиной (корнем) и $(l+1)$ выходными вершинами (листьями, которые занумерованы слева направо числами $1, \ldots, l+1)$ такой, что функция проводимости между корнем и $i$-м, $i=1, \ldots, l+1$, листом имеет вид

$$
f_{i}\left(u_{1}, v_{1}, u_{2}, v_{2}\right)= \begin{cases}1, & \text { если } z_{i-1} \leqslant v_{1}<z_{i} \\ 0 & \text { в противном случае }\end{cases}
$$

где $z_{0}=b, z_{l+1}=e$.

Если считать, что $i$-му листу, $i=1, \ldots, l+1$, соответствует точка $z_{i-1}$, то $U_{Z, e, f}^{2}$ позволяет находить точку из множества $\left\{b=z_{0}, z_{1}, \ldots, z_{l}\right\}$, ближайшую слева к $v_{1}$.

ИГ $U_{Z, e, f}^{2}$ строится аналогично ИГ $U_{Z, e, f}^{1}$ с помощью функций из $G_{2} \cup G_{4}$.

Вероятностная мера на множестве запросов $v_{1} \in[e, f]$ при условии, что $0 \leqslant b \leqslant u_{1} \leqslant d<1$ и $b \leqslant e$, в рассмотренной задаче о близости определяется функцией плотности вероятности, задаваемой соотношением (11). Поскольку справедливо (14), мы находимся в условиях теоремы 7 из [27]. Поэтому при $m=$ ] $c l[$

$$
\left.Q\left(U_{Z, e, f}^{2}\right)=(2+c) l+1, \quad T\left(U_{Z, e, f}^{2}\right) \leqslant 2, \quad T\left(U_{Z, e, f}^{2}, x\right) \leqslant 1+\right] \log _{2}(l+1)[
$$

для любого $x \in[e, f]$.

Пусть $Z=\left\{z_{1}, z_{2}, \ldots, z_{l}\right\}$ - упорядоченное множество действительных чисел из интервала $[0,1)$. Через $U_{Z}^{3}$ обозначим ИГ с одной входной вершиной (корнем) и $l$ выходными вершинами такой, что функция проводимости между корнем и листом с номером $i, i=1, \ldots, l$, имеет вид

$$
f_{i}\left(u_{1}, v_{1}, u_{2}, v_{2}\right)= \begin{cases}1, & \text { если } u_{2} \leqslant z_{i} \leqslant v_{2}, \\ 0 & \text { в противном случае. }\end{cases}
$$

Если считать, что $i$-му листу, $i=1, \ldots, l$, соответствует точка $z_{i}$, то $U_{Z}^{3}$ позволяет находить все точки из $Z$, принадлежащие отрезку $\left[u_{2}, v_{2}\right]$.

ИГ $U_{Z}^{3}$ разрешает одномерную задачу интервального поиска для библиотеки $V=Z$ и множества запросов $X=X_{\text {int }}$. ИГ $U_{Z}^{3}$ будем строить по методу, описанному 
впараграфе 2.4.1 в [27], с помощью функций из $G_{5} \cup G_{6}, F_{2}$. Неформальное описание алгоритма, соответствующего ИГ $U_{Z}^{3}$ (алгоритма решения одномерной задачи интервального поиска), приведено в предыдущем разделе.

Если для некоторых $b, d, e, f \in[0,1)$ таких, что $b<d \leqslant e<f$ выполняются неравенства $b \leqslant u_{1} \leqslant d$ и $e \leqslant v_{1} \leqslant f$, то вероятностная мера на множестве запросов определяется функцией плотности вероятности, задаваемой соотношением (9). Поскольку выполняется (12), мы находимся в условиях теоремы 8 из [27]. Следовательно, если в качестве параметра алгоритма (см. предыдущий раздел) выбрать $m=2 c\left[\log _{2} l\right]$, то

$$
Q\left(U_{Z}^{3}\right) \leqslant 4 l-1+6 c\left[\log _{2} l\right], \quad T\left(U_{Z}^{3}\right) \leqslant 5+\sum_{z \in Z} \mathbf{P}^{\prime}\left(O\left(z, \rho_{\text {int }}\right)\right)
$$

где $\mathbf{P}^{\prime}$ определяется функцией плотности вероятности (9), и для любого $\tilde{x} \in X_{\text {int }}$

$$
\left.T\left(U_{Z}^{3}, \tilde{x}\right) \leqslant 1+\right] \log _{2} l\left[+\left|\left\{z \in Z: \tilde{x} \rho_{\text {int }} z\right\}\right| .\right.
$$

Приступим к построению информационного графа $U^{*}$, решающего двумерную задачу интервального поиска методом, описанным в предыдущем разделе.

Сначала построим ИГ $U_{q}, q=1, \ldots, M$.

Все полюсы графа $U_{q}, q=1, \ldots, M$, разделяются на несколько типов.

(1) При $1 \leqslant q<M$ в графе $U_{q}$ есть полюсы $\vartheta_{j}^{q}, j=2, \ldots, k^{\alpha_{1}+\ldots \alpha_{q}}$, на которых реализуются функции

$$
f_{j}^{\vartheta, q}(\tilde{x})= \begin{cases}1, & \text { если } l_{j-1}^{q}<u_{1} \leqslant l_{j}^{q} \leqslant v_{1}, \\ 0 & \text { в противном случае. }\end{cases}
$$

Из вершин $\vartheta_{j}^{q}$ в дальнейшем будут выпускаться ИГ, находящие в множестве $\mathcal{N}_{j-1}^{q+1}$ точку, ближайшую справа к $u_{1}$.

(2) При $1 \leqslant q \leqslant M$ в $U_{q}$ есть полюса $\beta_{j}^{q}, j=1, \ldots, k^{\alpha_{1}+\ldots+\alpha_{q}}$, на которых реализуются функции

$$
f_{j}^{\beta, q}(\tilde{x})= \begin{cases}1, & \text { если } u_{1} \leqslant l_{j}^{q} \leqslant v_{1}<l_{j+1}^{q}, \\ 0 & \text { в противном случае. }\end{cases}
$$

При $q<M$ из вершин $\beta_{j}^{q}$ в дальнейшем будут выпускаться ИГ, находящие в множестве $\mathcal{N}_{j}^{q+1}$ точку, ближайшую слева к $v_{1}$, а при $q=M$ в этих вершинах будет выполняться проверка принадлежности отрезку $\left[u_{2}, v_{2}\right]$ ординаты точки библиотеки $V$ с абсциссой $l_{j}^{q}$.

(3) При $1 \leqslant q<M$ в $U_{q}$ есть полюсы $\gamma_{j}^{q}, j=2, \ldots, k^{\alpha_{1}+\ldots+\alpha_{q}}+1$. На них реализуются функции

$$
f_{j}^{\gamma, q}(\tilde{x})= \begin{cases}1, & \text { если } l_{j-1}^{q}<u_{1} \leqslant v_{1}<l_{j}^{q} \\ 0 & \text { в противном случае. }\end{cases}
$$

Из вершин $\gamma_{j}^{q}$ будут выпускаться ИГ, которые находят ближайшую справа к $u_{1}$ и ближайшую слева к $v_{1}$ точки при условии, что $u_{1}, v_{1} \in\left(l_{j-1}^{q}, l_{j}^{q}\right)$. 
(4) При $1 \leqslant q \leqslant M$ в $U_{q}$ есть полюсы $\delta_{s i j}^{q}, s=1, \ldots, k^{\alpha_{1}+\ldots+\alpha_{q-1}}, i=2, \ldots, k^{\alpha_{q}}-1$, $j=i+1, \ldots, k^{\alpha_{q}}$. При $q=1$ индексы меняются как $s=1, i=1, \ldots, k^{\alpha_{1}}-1$, $j=i+1, \ldots, k^{\alpha_{1}}$. На них реализуются функции

$f_{s i j}^{\delta, q}(\tilde{x})= \begin{cases}1, & l_{(s-1) k^{\alpha_{q}+i-1}}^{q}<u_{1} \leqslant l_{(s-1) k^{\alpha_{q}+i}}^{q}, l_{(s-1) k^{\alpha_{q}+j}}^{q} \leqslant v_{1}<l_{(s-1) k^{\alpha}+j+1}^{q}, \\ 0 & \text { в противном случае. }\end{cases}$

Из вершин $\delta_{s i j}^{q}$ будут выпускаться ИГ, решающие одномерную задачу интервального поиска для ординат тех точек из библиотеки $V$, абсциссы которых принадлежат интервалу $\left[l_{(s-1) k^{\alpha_{q}+i}}^{q}, l_{(s-1) k^{\alpha_{q}+j}}^{q}\right)$.

(5) При $1<q \leqslant M$ в $U_{q}$ есть полюсы $\eta_{i j}^{q}, i=2, \ldots, k^{\alpha_{1}+\ldots+\alpha_{q-1}}, j=1, \ldots, k^{\alpha_{q}}-1$, на которых реализуются функции

$$
f_{i j}^{\eta, q}(\tilde{x})= \begin{cases}1, & \text { если } l_{(i-2) k^{\alpha_{q}}+j}^{q}<u_{1} \leqslant l_{(i-2) k^{\alpha_{q}+j+1}}^{q}, \quad v_{1} \geqslant l_{i}^{q-1}, \\ 0 & \text { в противном случае. }\end{cases}
$$

Из вершин $\eta_{i j}^{q}$ будут в дальнейшем выпускаться ИГ, решающие одномерную задачу интервального поиска для ординат тех точек библиотеки $V$, абсциссы которых принадлежат интервалу $\left[l_{(i-2) k^{\alpha}+j+1}^{q}, l_{i}^{q-1}\right)$.

(6) При $1<q \leqslant M$ в $U_{q}$ есть полюсы $\zeta_{i j}^{q}, i=1, \ldots, k^{\alpha_{1}+\ldots+\alpha_{q-1}}, j=2, \ldots, k^{\alpha_{q}}$, на которых реализуются функции

$$
f_{i j}^{\zeta, q}(\tilde{x})= \begin{cases}1, & \text { если } l_{(i-1) k^{\alpha_{q}}+j}^{q} \leqslant v_{1}<l_{(i-1) k^{\alpha_{q}}+j+1}^{q}, \quad u_{1} \leqslant l_{i}^{q-1}, \\ 0 & \text { в противном случае. }\end{cases}
$$

Из вершин $\zeta_{i j}^{q}$ будут в дальнейшем выпускаться ИГ, решающие одномерную задачу интервального поиска для ординат тех точек библиотеки $V$, абсциссы которых принадлежат интервалу $\left[l_{i}^{q-1}, l_{(i-1) k^{\alpha_{q}+j}}^{q}\right)$.

Строить $U_{q}, q=1, \ldots, M$, будем индуктивно по $q$.

Пусть $q=1$. Возьмем ИГ $U_{\mathcal{N}^{1}, 0,1}^{1}$. Он имеет $\left(k^{\alpha_{1}}+1\right)$ концевых вершин. Обозначим их $\theta_{1}, \ldots, \theta_{k^{\alpha_{1}}+1}$.

Если $M>1$, то для вершины $\theta_{k^{a_{1}+1}}$ введем новое обозначение $\gamma_{k^{\alpha_{1}}+1}^{1}$, так как мы попадаем в нее только в том случае, когда

$$
l_{k^{\alpha_{1}}}^{1}<u_{1} \leqslant v_{1}<l_{k^{\alpha_{1}}+1}^{1}=1 .
$$

Если $M=1$, то пометим ее символом *. Выход к вершине, помеченной символом * будет означать, что ответ на запрос $\tilde{x}=\left(u_{1}, v_{1}, u_{2}, v_{2}\right)$ пуст. Эта метка делается только для наглядности описания, на функционирование ИГ она не влияет.

Из каждой вершины $\theta_{i}, i=1, \ldots, k^{\alpha_{1}}$, выпустим два ребра, припишем левому 1 , правому 2, а самой вершине $\theta_{i}$ припишем переключатель $g_{l_{i}^{\mathbf{4}}}^{\mathbf{4}}(\tilde{x}) \in G^{4}$, обозначим конец левого ребра $\theta_{i}^{\prime}$, конец правого $\theta_{i}^{\prime \prime}$. В $\theta_{i}^{\prime}$ мы попадаем только в том случае, когда

$$
l_{i-1}^{1}<u_{1} \leqslant v_{1}<l_{i}^{1},
$$




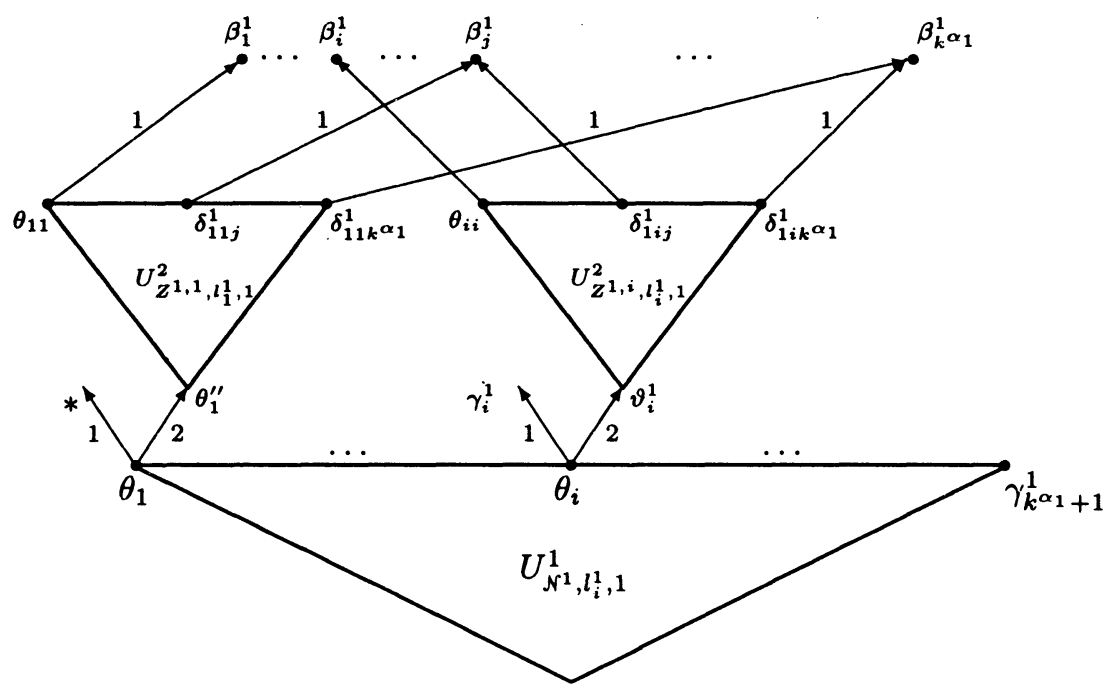

Рис. 3. Информационный граф $U_{1}, M>1$

а в $\theta_{i}^{\prime \prime}$, когда $l_{i-1}^{1}<u_{1} \leqslant l_{i}^{1}, v_{1} \geqslant l_{i}^{1}$. Поэтому если $M>1$, то мы можем обозначить все $\theta_{i}^{\prime}$ как в $\gamma_{i}^{1}$, а $\theta_{i}^{\prime \prime}$ как $\vartheta_{i}^{1}, i=2, \ldots, k^{\alpha_{1}}$.

Вершину $\theta_{1}^{\prime}$ пометим символом *.

Если $M=1$, то все $\theta_{i}^{\prime}, i=1, \ldots, k^{\alpha_{1}}$, пометим символом $*$.

Каждую вершину $\theta_{i}^{\prime \prime}, i=1, \ldots, k^{\alpha_{1}}-1$ (включая переобозначенные в $\vartheta_{i}^{1}$ ), отождествим с корнем ИГ $U_{Z^{1, i}, l_{i}^{1}, 1}^{2}$, где

$$
Z^{1, i}=\left\{l_{j}^{1} \in \mathcal{N}^{1} \mid j \geqslant i+1\right\} .
$$

Информационный граф $U_{Z^{1, i}, l_{i}^{1}, 1}^{2}$ имеет $k^{\alpha_{1}}-i+1$ концевых вершин, которые обозначим $\theta_{i j}, i=1, \ldots, k^{\alpha_{1}}-1, j=i, \ldots, k^{\alpha_{1}}$. Понятно, что в вершины $\theta_{i j}$ мы проходим только в том случае, когда

$$
l_{i-1}^{1}<u_{1} \leqslant l_{i}^{1}, \quad l_{j}^{1} \leqslant v_{1}<l_{j+1}^{1} .
$$

Следовательно, можно переобозначить вершины $\theta_{i j}, i=1, \ldots, k^{\alpha_{1}}-1, j=$ $i+1, \ldots, k^{\alpha_{1}}$, в $\delta_{1 i j}^{1}$.

Введем новые вершины $\beta_{j}^{1}, j=1, \ldots, k^{\alpha_{1}}$, и из каждой $\theta_{i j}, i=1, \ldots, j$ (включая $\theta_{i j}$, переобозначенные в $\delta_{1 i j}^{1}$ ), проведем ребро в вершину $\beta_{j}^{1}$, которому припишем предикат, тождественно равный 1. Очевидно, что

$$
\varphi_{\beta_{j}^{1}}= \begin{cases}1, & \text { если } l_{j}^{1} \leqslant v_{1}<l_{j+1}^{1} \text { и } u_{1} \leqslant l_{j}^{1}, \\ 0 & \text { в противном случае }\end{cases}
$$

то есть $\varphi_{\beta_{j}^{1}}=f_{j}^{\beta .1}(\tilde{x})$.

Построенный граф обозначим $U_{1}$. Он изображен на рис. 3 для случая $M>1$.

Опишем теперь индуктивный переход. Пусть построен ИГ $U_{q-1}$. Будем строить $U_{q}$, достраивая $U_{q-1}$ следующим образом. 
Из каждой вершины $\vartheta_{i}^{q-1}, i=2, \ldots, k^{\alpha_{1}+\ldots+\alpha_{4-1}}$, выпустим информационный граф $U_{\mathcal{N}_{i-1}^{q}, l_{i-1}^{q-1}, l_{i}^{q-1}}^{1}$. Этот граф имеет $k^{\alpha_{q}}$ листьев, которые обозначим $\tau_{q i j}^{\prime}, j=$ $1, \ldots, k^{\alpha_{q}}$.

В вершину $\tau_{q i j}^{\prime}$ мы попадаем только в том случае, когда

$$
l_{(i-2) k^{\alpha_{q}}+j}^{q}<u_{1} \leqslant l_{(i-2) k^{\alpha_{q}}+j+1}^{q}, \quad v_{1} \geqslant l_{i}^{q-1},
$$

поскольку

$$
\mathcal{N}_{i-1}^{q}=\left(l_{i-1}^{q-1}, l_{i}^{q-1}\right)=\left(l_{(i-2) k^{\alpha}+1}^{q}, l_{(i-1) k^{\alpha+1}}^{q}\right) .
$$

Получаем, что $\varphi_{\tau_{q i j}^{\prime}}=f_{i j}^{\eta, q}(\tilde{x})$. Следовательно, можно переобозначить $\tau_{q i j}^{\prime}$ с индексами $i=2, \ldots, k^{\alpha_{1}+\ldots+\alpha_{q-1}}, j=1, \ldots, k^{\alpha_{q}}-1$, в $\eta_{i j}^{q}$.

Из каждой вершины $\beta_{i}^{q-1}, i=1, \ldots, k^{\alpha_{1}+\ldots+\alpha_{q-1}}$, выпустим информационный граф $U_{\mathcal{N}_{i}^{q}, l_{i}^{q-1}, l_{i+1}^{q-1}}^{2}$. Граф имеет $k^{\alpha_{q}}$ листьев, которые обозначим $\tau_{q i j}^{\prime \prime}, j=1, \ldots, k^{\alpha_{q}}$ В вершину $\tau_{q i j}^{\prime \prime}$ мы попадаем только в том случае, когда

$$
l_{(i-1) k^{\alpha_{q}}+j}^{q} \leqslant v_{1}<l_{(i-1) k^{\alpha}+j+1}^{q}, \quad u_{1} \leqslant l_{i}^{q-1} .
$$

Таким образом, $\varphi_{\tau_{q i j}^{\prime \prime}}=f_{i j}^{\zeta, q}(\tilde{x})$. Следовательно, можно переобозначить $\tau_{q i j}^{\prime \prime}$ с индексами $i=1, \ldots, k^{\alpha_{1}+\ldots+\alpha_{q-1}}, j=2, \ldots, k^{\alpha_{q}}$, в $\zeta_{i j}^{q}$.

Рассмотрим вершины $\gamma_{i}^{q-1}, i=2, \ldots, k^{\alpha_{1}+\ldots+\alpha_{q-1}}+1$. Из каждой такой вершины выпустим информационный граф $U_{\mathcal{N}_{i-1}^{q}, l_{i-1}^{q-1}, l_{i}^{q-1}}^{1}$. Этот граф имеет $k^{\alpha_{q}}$ листьев. Обозначим их $\theta_{q i j}, j=1, \ldots, k^{\alpha_{q}}$. Проводимость в эти вершины будет равна

$$
\varphi_{\theta_{q i j}}= \begin{cases}1, & \text { если } l_{(i-2) k^{\alpha_{q}+j}}^{q}<u_{1} \leqslant l_{(i-2) k^{\alpha_{q}+j+1}}^{q}, v_{1}<l_{(i-1) k^{\alpha_{q}+1}}^{q}, \\ 0 & \text { в противном случае. }\end{cases}
$$

Из каждой вершины $\theta_{q i j}, i=2, \ldots, k^{\alpha_{1}+\ldots+\alpha_{q-1}}+1, j=1, \ldots, k^{\alpha_{q}}-1$, выпустим два ребра, припишем левому 1 , правому 2 , а самой $\theta_{q i j}$ припишем переключа-

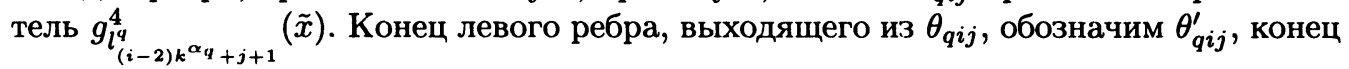
правого $\theta_{q i j}^{\prime \prime}$. Переобозначим все $\theta_{q, i, k^{\alpha_{q}}}, i=2, \ldots, k^{\alpha_{1}+\ldots+\alpha_{q-1}}+1$, в $\theta_{q, i, k^{\alpha_{q}}}^{\prime}$.

Если $q<M$, то переобозначим все $\theta_{q i j}^{\prime}, i=2, \ldots, k^{\alpha_{1}+\ldots+\alpha_{q-1}}+1, j=1, \ldots, k^{\alpha_{q}}$, в $\gamma_{(i-2) k^{\alpha}+j+1}^{q}$, так как в $\theta_{q i j}^{\prime}$ мы можем попасть только в том случае, когда

$$
l_{(i-2) k^{\alpha_{q}+j}}^{q}<u_{1} \leqslant v_{1}<l_{(i-2) k^{\alpha_{q}}+j+1}^{q},
$$

то есть $\varphi_{\theta_{q i j}^{\prime}}=f_{(i-2) k^{\alpha} q+j+1}^{\gamma, q}(\tilde{x})$.

При изменении $i$ от 2 до $k^{\alpha_{1}+\ldots+\alpha_{q-1}}+1, j$ от 1 до $k^{\alpha_{q}},(i-2) k^{\alpha_{q}}+j+1$ изменяется от 2 до $k^{\alpha_{1}+\ldots+\alpha_{q}}+1$, поэтому мы получим все вершины типа $\gamma_{s}^{q}$, $s=2, \ldots, k^{\alpha_{1}+\ldots+\alpha_{u}}+1$.

Если $q=M$, то все $\theta_{q i j}^{\prime}, i=2, \ldots, k^{\alpha_{1}+\ldots+\alpha_{q-1}}+1, j=1, \ldots, k^{\alpha_{q}}$, пометим символом $*$.

В вершины $\theta_{q i j}^{\prime \prime}$ мы попадаем при условии, что

$$
l_{(i-2) k^{\alpha_{q}}+j}^{q}<u_{1} \leqslant l_{(i-2) k^{\alpha_{q}+j+1}}^{q} \leqslant v_{1}<l_{(i-1) k^{\alpha_{q}+1}}^{q} .
$$


Из каждой вершины $\theta_{q i j}^{\prime \prime}, i=2, \ldots, k^{\alpha_{1}+\ldots+\alpha_{q-1}}+1, j=1, \ldots, k^{\alpha_{q}}-2$, выпустим

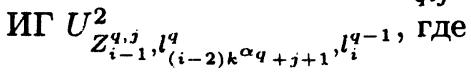

$$
Z_{i-1}^{q, j}=\left\{l_{(i-2) k^{\alpha_{q}}+j+2}^{q}, \ldots, l_{(i-1) k^{\alpha_{q}}}^{q}\right\}
$$

Этот информационный граф имеет $\left(k^{\alpha_{q}}-j\right)$ листьев, которые обозначим

$$
\mu_{i j t}^{q}, \quad i=2, \ldots, k^{\alpha_{1}+\ldots+\alpha_{q-1}}+1, \quad j=1, \ldots, k^{\alpha_{q}}-2, \quad t=j+1, \ldots, k^{\alpha_{q}} .
$$

Вершины $\theta_{q, i, k^{\alpha} q-1}^{\prime \prime}$ переобозначим в $\mu_{i, k^{\alpha_{q}}-1, k^{\alpha_{q}}}^{q}$

В вершину $\mu_{i j t}^{q}$ мы проходим только в том случае, когда

$$
l_{(i-2) k^{\alpha_{q}}+j}^{q}<u_{1} \leqslant l_{(i-2) k^{\alpha_{q}}+j+1}^{q}, \quad l_{(i-2) k^{\alpha_{q}+t}}^{q} \leqslant v_{1}<l_{(i-2) k^{\alpha_{q}}+t+1}^{q} .
$$

Следовательно, $\varphi_{\mu_{i j t}^{q}}=f_{i-1, j+1, t}^{\delta, q}(\tilde{x})$, и поэтому можно переобозначить вершины $\mu_{i j t}^{q}$, $i=2, \ldots, k^{\alpha_{1}+\ldots+\alpha_{q-1}}+1, j=1, \ldots, k^{\alpha_{q}}-2, t=j+2, \ldots, k^{\alpha_{q}}$, в $\delta_{i-1, j+1, t}^{q}$.

Так как $i-1$ изменяется от 1 до $k^{\alpha_{1}+\ldots+\alpha_{q-1}}, j+1$ изменяется от 2 до $k^{\alpha_{q}}-1$, $t$ изменяется от $(j+1)+1$ до $k^{\alpha_{q}}$, мы получаем все нужные вершины типа $\delta_{s p r}^{q}$, $s=1, \ldots, k^{\alpha_{1}+\ldots+\alpha_{q-1}}, p=2, \ldots, k^{\alpha_{q}}-1, r=p+1, \ldots, k^{\alpha_{q}}$.

Если $q<M$, то введем новые вершины $\vartheta_{t}^{q}, t=2, \ldots, k^{\alpha_{1}+\ldots+\alpha_{q}}$. Из каждой вершины $\theta_{q i j}^{\prime \prime}, i=2, \ldots, k^{\alpha_{1}+\ldots+\alpha_{q-1}}+1, j=1, \ldots, k^{\alpha_{q}}-1$, и каждой вершины $\tau_{q i j}^{\prime}$, $i=2, \ldots, k^{\alpha_{1}+\ldots+\alpha_{q-1}}, j=1, \ldots, k^{\alpha_{q}}$ (включая переобозначенные в $\eta_{i j}^{q}$ ), проведем ребро в вершину $\vartheta_{(i-2) k^{\alpha_{4}+j+1}}^{q}$, которому припишем предикат, тождественно равный единице. Поскольку проводимость в вершины $\theta_{q i j}^{\prime \prime}$ определяется соотношением (31), а в вершины $\tau_{q i j}^{\prime}$ формулой $(24)$, на $\vartheta_{(i-2) k^{\alpha_{q}+j+1}}^{q}$ мы имеем нужную нам функцию, так каK

$$
\varphi_{\vartheta_{(i-2) k^{\alpha} q}^{q}+j+1}= \begin{cases}1, & \text { если } l_{(i-2) k^{\alpha} q+j}^{q}<u_{1} \leqslant l_{(i-2) k^{\alpha} q+j+1}^{q} \leqslant v_{1}, \\ 0 & \text { в противном случае. }\end{cases}
$$

При изменении $i$ от 2 до $k^{\alpha_{1}+\ldots+\alpha_{q-1}}+1, j$ от 1 до $k^{\alpha_{q}}, t=(i-2) k^{\alpha_{q}}+j+1$ изменяется от 2 до $k^{\alpha_{1}+\ldots+\alpha_{4}}$, то есть в нужных нам пределах. Здесь индекс $k^{\alpha_{1}+\ldots+\alpha_{4}}+1$ не появляется поскольку при $i=k^{\alpha_{1}+\ldots+\alpha_{q-1}}+1 j$ меняется от 1 до $k^{\alpha_{q}}-1$.

Введем новые вершины $\beta_{s}^{q}, s=1, \ldots, k^{\alpha_{1}+\ldots+\alpha_{q}}$. Из каждой вершины $\mu_{i j t}^{q}$, $i=2, \ldots, k^{\alpha_{1}+\ldots+\alpha_{q-1}}+1, j=1, \ldots, k^{\alpha_{4}}-1, t=j+1, \ldots, k^{\alpha_{q}}$ (включая вершины, переобозначенные в $\left.\delta_{i-1, j+1, t}^{q}\right)$, выпустим ребро в вершину $\beta_{(i-2) k^{\alpha}+t}^{q}$ и из каждой вершіны $\tau_{q i j}^{\prime \prime}, i=1, \ldots, k^{\alpha_{1}+\ldots+\alpha_{q-1}}, j=1, \ldots, k^{\alpha_{q}}$ (включая переобозначенные в $\left.\zeta_{i j}^{q}\right)$, выпустим ребро в вершину $\beta_{(i-1) k^{\alpha_{q}+j}}^{q}$, ребрам припишем предикаты, тождественно равные единице.

Учитывая соотношения (29), (32), легко заметить, что функция фильтра на введенных вершинах $\beta_{s}^{q}$ совпадает с нужной нам функцией, задаваемой соотношением $(21)$, и $s$ изменяется в пределах от 1 до $k^{\alpha_{1}+\ldots+\alpha_{q}}$.

Тем самым построение ИГ $U_{q}$ завершено.

Пусть мы построили информационный граф $U_{M}$. Теперь, чтобы завершить построение окончательного ИГ $U^{*}$, решающего задачу нашим методом, надо добавить фрагменты, решающие одномерные задачи интервального поиска по вторым координатам $\left(u_{2}, v_{2}\right)$. 
Пусть $q, s, i, j$ - натуральные числа, $q \in\{1, \ldots, M\}, s \in\left\{1, \ldots, k^{\alpha_{1}+\ldots+\alpha_{q-1}}\right\}$, $i \in\left\{1, \ldots, k^{a_{q}}\right\}, j \in\left\{i+1, \ldots, k^{a_{q}}+1\right\}$, введем обозначения

$$
A_{s, i, j}^{q}=\left\{y_{p}^{2}:\left(y_{p}^{1}, y_{p}^{2}\right) \in V, l_{(s-1) k^{\alpha_{q}+i}}^{q} \leqslant y_{p}^{1}<l_{(s-1) k^{\alpha_{q}+j}}^{q}\right\}
$$

то есть $A_{s, i, j}^{q}$ состоит из ординат точек библиотеки, абсциссы которых принадлежат интервалу $\left[l_{(s-1) k^{\alpha_{4}+i}}^{q}, l_{(s-1) k^{\alpha_{q}+j}}^{q}\right)$.

ИГ $U_{M}$ содержит полюсы

$\delta_{s i j}^{q}, \quad q=1, \ldots, M, \quad s=1, \ldots, k^{\alpha_{1}+\ldots+\alpha_{q-1}}, \quad i=2, \ldots, k^{\alpha_{q}}-1, \quad j=i+1, \ldots, k^{\alpha_{q}}$.

При $q=1$ индексы меняются так: $s=1, i=1, \ldots, k^{\alpha_{1}}-1, j=i+1, \ldots, k^{\alpha_{1}}$. В эти полюсы мы попадаем при условии, что

$$
l_{(s-1) k^{\alpha_{q}+i-1}}^{q}<u_{1} \leqslant l_{(s-1) k^{\alpha_{q}+i}}^{q}, \quad l_{(s-1) k^{\alpha_{q}+j}}^{q} \leqslant v_{1}<l_{(s-1) k^{\alpha_{q}+j+1}}^{q} .
$$

Из каждого полюса $\delta_{s i j}^{q}$ выпустим ИГ $U_{A_{s, i, j}^{q}}^{3}$, который решает одномерную задачу интервального поиска для запроса $\left(u_{2}, v_{2}\right)$ среди точек из $A_{s, i, j}^{q}$. Листья ИГ $U_{A_{s, i, j}^{q}}^{3}$, которым соответствуют точки $y_{p}^{2} \in A_{s, i, j}^{q}$, мы объявим листьями окончательного ИГ $U^{*}$ и припишем им записи $\left(y_{p}^{1}, y_{p}^{2}\right) \in V$.

ИГ $U_{M}$ содержит полюсы

$$
\eta_{i j}^{q}, \quad q=2, \ldots, M, \quad i=2, \ldots, k^{\alpha_{1}+\ldots+\alpha_{q-1}}, \quad j=1, \ldots, k^{\alpha_{q}}-1,
$$

в которые мы попадаем при условии, что

$$
l_{(i-2) k^{\alpha_{q}}+j}^{q}<u_{1} \leqslant l_{(i-2) k^{\alpha_{q}+j+1}}^{q}, \quad v_{1} \geqslant l_{(i-1) k^{\alpha_{q}}+1}^{q} .
$$

Из каждого полюса $\eta_{i j}^{q}$ выпустим ИГ $U_{A_{i-1, j+1, k^{\alpha} q+1}^{3}}^{3}$. Листья ИГ $U_{A_{i-1, j+1, k^{\alpha}+1}^{\alpha_{q}}}^{3}$, которым соответствуют точки $y_{p}^{2} \in A_{i-1, j+1, k^{\alpha}+1}^{q}$, мы объявим листьями окончательного ИГ $U^{*}$ и припишем им записи $\left(y_{p}^{1}, y_{p}^{2}\right) \in V$.

ИГ $U_{M}$ содержит полюсы $\zeta_{i j}^{q}, q=2, \ldots, M, i=1, \ldots, k^{\alpha_{1}+\ldots+\alpha_{q-1}}, j=2, \ldots, k^{\alpha_{q}}$, в которые мы попадаем при условии, что

$$
u_{1} \leqslant l_{(i-1) k^{\alpha_{q}}+1}^{q}, \quad l_{(i-1) k^{\alpha_{q}}+j}^{q} \leqslant v_{1}<l_{(i-1) k^{\alpha_{q}}+j+1}^{q} .
$$

Из каждого полюса $\zeta_{i j}^{q}$ выпустим ИГ $U_{A_{i, 1, j}^{y}}^{3}$. Листья ИГ $U_{A_{i, 1, j}^{q}}^{3}$, которым соответствуют точки $y_{p}^{2} \in A_{i, 1, j}^{q}$, мы объявим листьями окончательного ИГ $U^{*}$ и припишем им записи $\left(y_{p}^{1}, y_{p}^{2}\right) \in V$.

ИГ $U_{M}$ содержит полюсы $\beta_{j}^{M}, j=1, \ldots, k^{\alpha_{1}+\ldots+\alpha_{M}}$, в которые мы попадаем при условии, что

$$
l_{j-1}^{M}<u_{1} \leqslant l_{j}^{M} \leqslant v_{1}<l_{j+1}^{M} .
$$

Из каждого полюса $\beta_{j}^{M}$ выпустим одно ребро, которому припишем предикат $f_{y_{j}^{2}} \in F_{2}$. Конец ребра объявим листом и припишем ему запись $\left(y_{j}^{1}, y_{j}^{2}\right) \in V$.

Полученный окончательный ИГ обозначим $U^{*}$. Он соответствует алгоритму решения двумерной задачи интервального поиска, описанному в предыдущем разделе. 


\section{5. Допустимость информационного графа}

Покажем, что ИГ $U^{*}$ решает ЗИП $I=\left\langle X_{\text {int 2 }}, V, \rho_{\text {int 2 }}\right\rangle$.

Возьмем произвольный запрос $\tilde{x}=\left(u_{1}, v_{1}, u_{2}, v_{2}\right)$. Возможны следующие случаи.

1. Для любого $q \in\{1, \ldots, M\}$ существует номер $r=r(q) \in\left\{1, \ldots, k^{\alpha_{1}+\ldots+\alpha_{q}}+1\right\}$ такой, что $l_{r-1}^{q}<u_{1} \leqslant v_{1}<l_{r}^{q}$. Это означает, что $\left\{\tilde{y} \in V: \tilde{x} \rho_{\text {int } 2} \tilde{y}\right\}=\varnothing$. Поскольку путь к листьям ИГ $U^{*}$ лежит только через вершины типов $\delta_{s i j}^{q}, \eta_{i j}^{q}, \zeta_{i j}^{q}, \beta_{j}^{M}$, а проводимость в эти вершины есть только в случаях, когда хотя бы одна точка сетки попадает в интервал $\left[u_{1}, v_{1}\right]$, проводимость в эти вершины на запросе $\tilde{x}$ равна нулю и ответ ИГ $U^{*}$ на запрос $\tilde{x}$ пуст.

2. Существуют такие номера $q \in\{1, \ldots, M\}$ и $r=r(q) \in\left\{1, \ldots, k^{\alpha_{1}+\ldots+\alpha_{q}}\right\}$, что $u_{1} \leqslant l_{r}^{q} \leqslant v_{1}$. Пусть $q_{0}-$ минимальный из номеров $q$, обладающих таким свойством, то есть либо $q_{0}=1$, либо для любого $q<q_{0}$ существует такой номер $r=r(q) \in\left\{1, \ldots, k^{\alpha_{1}+\ldots+\alpha_{q}}+1\right\}$, что $l_{r-1}^{q}<u_{1} \leqslant v_{1}<l_{r}^{q}$. Возможны два подслучая.

2.1. Для любого $q \in\left\{q_{0}, \ldots, M\right\}$ существует номер $r=r(q) \in\left\{1, \ldots, k^{\alpha_{1}+\ldots+\alpha_{q}}\right\}$ такой, что $l_{r-1}^{q}<u_{1} \leqslant l_{r}^{q} \leqslant v_{1}<l_{r+1}^{q}$. Это означает, что множество $\left\{\tilde{y} \in V: \tilde{x} \rho_{\text {int } 2} \tilde{y}\right\}$ содержит не более одной записи, причем единственным кандидатом на принадлежность этому множеству является запись $\left(y_{r(M)}^{1}, y_{r(M)}^{2}\right)$. Если $u_{2} \leqslant y_{r(M)}^{2} \leqslant v_{2}$, то $\left(y_{r(M)}^{1}, y_{r(M)}^{2}\right) \in\left\{\tilde{y} \in V: \tilde{x} \rho_{\text {int } 2} \tilde{y}\right\}$, иначе $\left\{\tilde{y} \in V: \tilde{x} \rho_{\text {int } 2} \tilde{y}\right\}=\varnothing$. Непосредственно проверяется, что среди вершин типа $\beta_{j}^{M}$ только проводимость в вершину $\beta_{r(M)}^{M}$ будет ненулевой, тогда как проводимость всех вершин типа $\delta_{s i j}^{q}, \eta_{i j}^{q}, \zeta_{i j}^{q}$ будет равна нулю, так как мы проходим в эти вершины только тогда, когда по крайней мере две точки сетки попадают в интервал $\left[u_{1}, v_{1}\right]$. Из вершины $\beta_{r(M)}^{M}$ исходит ребро с предикатом $f_{y_{r(M)}^{2}}$, что соответствует проверке принадлежности интервалу $\left[u_{1}, v_{1}\right]$ точки $y_{r(M)}^{2}$. Таким образом $\mathscr{F}_{U^{*}}(\tilde{x})=\left\{\tilde{y} \in V: \tilde{x} \rho_{\text {int } 2} \tilde{y}\right\}$.

2.2. Существуют номера $q \in\left\{q_{0}, \ldots, M\right\}$ и $r=r(q) \in\left\{1, \ldots, k^{\alpha_{1}+\ldots+\alpha_{q}}-1\right\}$ такие, что $u_{1} \leqslant l_{r}^{q} \leqslant l_{r+1}^{q} \leqslant v_{1}$. Пусть $q_{1}-$ минимальный из номеров $q$, обладающих таким свойством, то есть либо $q_{1}=q_{0}$, либо для любого $q$ такого, что $q_{0} \leqslant q<q_{1}$, существует такой номер $r=r(q) \in\left\{1, \ldots, k^{\alpha_{1}+\ldots+\alpha_{4}}\right\}$, что

$$
l_{r-1}^{q}<u_{1} \leqslant l_{r}^{q} \leqslant v_{1}<l_{r+1}^{q}
$$

Для каждого $q \in\{1, \ldots, M\}$ определим такие номера $r^{\prime}(q)$ и $r^{\prime \prime}(q)$, что $l_{r^{\prime}(q)-1}^{q}<u_{1} \leqslant l_{r^{\prime}(q)}^{q}$ и $l_{r^{\prime \prime}(q)}^{q} \leqslant v_{1}<l_{r^{\prime \prime}(q)+1}^{q}$. Понятно, что если $q_{0}>1$, то для любого $q \in\left\{1, \ldots, q_{0}-1\right\}$ справедливо равенство $r^{\prime}(q)=r^{\prime \prime}(q)-1$, и если $q_{0}<q_{1}$, то для любого $q \in\left\{q_{0}, \ldots, q_{1}-1\right\}$ справедливо равенство $r^{\prime}(q)=r^{\prime \prime}(q)$, и для любого $q \in\left\{q_{1}, \ldots, M\right\}$ справедливо неравенство $r^{\prime}(q)<r^{\prime \prime}(q)$. Отметим, что если $q_{1}>1$, то для $q<q_{1}$ согласно сделанному выше замечанию проводимость всех вершин типа $\delta_{s i j}^{q}, \eta_{i j}^{q}, \zeta_{i j}^{q}$ будет равна нулю.

Заметим, что для того, чтобы проводимость в вершины типа $\delta_{s i j}^{q}$ была равна единице, необходимо, чтобы $q=1$, либо $u_{1}$ и $v_{1}$ принадлежали одному интервалу $(q-1)$-й сетки. Поэтому, если $q_{0}<q_{1}$, то проводимость во все вершины типа $\delta_{s i j}^{q}$, будет равна 0 . Если же $q_{0}=q_{1}$, то среди вершин типа $\delta_{s i j}^{q}$ только проводимость вершины $\delta_{s^{\prime}, i^{\prime}, j^{\prime}}^{q_{1}}$ будет равна 1 , где $s^{\prime}=1$, если $q_{1}=1$, и $s^{\prime}=r^{\prime \prime}\left(q_{1}-1\right)$, если $q_{1}>1$, $i^{\prime}=r^{\prime}(q)-\left(s^{\prime}-1\right) k^{\alpha_{q}}, j^{\prime}=r^{\prime \prime}(q)-\left(s^{\prime}-1\right) k^{\alpha_{u}}$. Выход к вершине $\delta_{s^{\prime}, i^{\prime}, j^{\prime}}^{q}$ означает, что будет решаться одномерная задача интервального поиска для запроса $\left(u_{2}, v_{2}\right)$ среди точек из множества $A_{s^{\prime}, i^{\prime}, j^{\prime}}^{q}$, определяемого соотношением (33). 
Легко видеть, что если $q_{0}=q_{1}$, то проводимость всех вершин типа $\eta_{i j}^{q_{1}}, \zeta_{i j}^{q_{1}}$ будет равна нулю.

Для каждого $q \in\left\{\max \left(q_{0}+1, q_{1}\right), \ldots, M\right\}$ среди вершин типа $\eta_{i j}^{q}$, проводимость только одной вершины $\eta_{i^{\prime \prime}(q), j^{\prime \prime}(q)}^{q}$ может быть равна 1 , и это случится при условии, что $r^{\prime}(q-1)>1$ и $r^{\prime}(q)<\left(r^{\prime}(q-1)-1\right) k^{\alpha_{q}}+1$, при этом $i^{\prime \prime}(q)=r^{\prime}(q-1)$ и $j^{\prime \prime}(q)=r^{\prime}(q)-\left(r^{\prime}(q-1)-2\right) k^{\alpha_{q}}-1$. Выход к вершине $\eta_{i^{\prime \prime}(q), j^{\prime \prime}(q)}$ означает, что будет решаться одномерная задача интервального поиска для запроса $\left(u_{2}, v_{2}\right)$ среди точек из множества $A_{i^{\prime \prime}(q)-1, j^{\prime \prime}(q)+1, k^{\alpha_{q}+1}}^{q}$.

Для каждого $q \in\left\{\max \left(q_{0}+1, q_{1}\right), \ldots, M\right\}$ среди вершин типа $\zeta_{i j}^{q}$ проводимость только одной вершины $\zeta_{i^{\prime \prime \prime}(q), j^{\prime \prime \prime}(q)}^{q}$ может быть равна 1 , и это случится при условии, что $r^{\prime \prime}(q)>\left(r^{\prime \prime}(q-1)-1\right) k^{\alpha_{q}}+1$ и при этом

$$
i^{\prime \prime \prime}(q)=r^{\prime \prime}(q-1), \quad j^{\prime \prime \prime}(q)=r^{\prime \prime}(q)-\left(r^{\prime \prime}(q-1)-1\right) k^{\alpha_{q}} .
$$

Выход к вершине $\zeta_{i^{\prime \prime \prime}(q), j^{\prime \prime \prime}(q)}^{q}$ означает, что будет решаться одномерная задача интервального поиска для запроса $\left(u_{2}, v_{2}\right)$ среди точек из множества $A_{i^{\prime \prime \prime}(q), 1, j^{\prime \prime \prime}(q)}^{q}$.

И наконец, среди вершин типа $\beta_{j}^{M}$, проводимость только вершины $\beta_{r^{\prime \prime}(M)}^{M}$ будет равна 1 , и в этой вершине делается проверка на принадлежность отрезку $\left[u_{2}, v_{2}\right]$ точки $y_{r^{\prime \prime}(M)}^{2}$.

Тем самым, легко видеть, что все множество записей из $V$, абсциссы которых принадлежат отрезку $\left[u_{1}, v_{1}\right]$, разделилось на непересекающиеся части и для каждой части решается одномерная задача интервального поиска по вторым координатам, то есть устанавливается принадлежность ординат записей отрезку $\left[u_{1}, v_{2}\right]$. A это означает, что ответ ИГ $U^{*}$ на запрос $\tilde{x}$ совпадает с множеством записей из $V$, удовлетворяющих запросу $\tilde{x}$.

Таким образом, в силу произвольности $\tilde{x}$ мы доказали допустимость ИГ $U^{*}$.

\section{6. Объем информационного графа}

Для оценки объема информационного графа $U^{*}$ вычислим сначала объем ИГ $U_{1}$.

ИГ $U_{1}$ содержит ИГ $U_{\mathcal{N}^{1}, 0,1}^{1}$, объем которого согласно (16) равен

$$
Q\left(U_{\mathcal{N}^{1}, 0,1}^{1}\right)=(2+c) k^{\alpha_{1}}+1
$$

Из каждой вершины $\tau_{i}, i=1, \ldots, k^{\alpha_{1}}$, исходит два ребра, что суммарно дает $2 k^{\alpha_{1}}$ ребер.

Из каждой вершины $\tau_{i}^{\prime \prime}, i=1, \ldots, k^{\alpha_{1}}-1$, исходит ИГ $U_{Z^{1, i}, l_{i}^{1}, 1}^{2}$, объем которого согласно (17) равен

$$
Q\left(U_{Z^{1, i}, l_{i}^{1}, 1}^{2}\right)=(2+c)\left(k^{\alpha_{1}}-i\right)+1
$$

В каждую вершину $\beta_{j}^{1}, j=1, \ldots, k^{\alpha_{1}}$, из каждой вершины $\tau_{i j}, i=1, \ldots, j$, ведет ребро, то есть суммарное число ребер, ведущих в вершины $\beta_{j}^{1}$, равно

$$
\sum_{j=1}^{k^{\alpha_{1}}} j=\frac{k^{\alpha_{1}}\left(k^{\alpha_{1}}+1\right)}{2}
$$


Таким образом,

$$
\begin{aligned}
Q\left(U_{1}\right)=(2+c) k^{\alpha_{1}}+1+2 k^{\alpha_{1}} \\
\\
\quad+\sum_{i=1}^{k^{\alpha_{1}}-1}\left((2+c)\left(k^{\alpha_{1}}-i\right)+1\right)+\frac{k^{\alpha_{1}}\left(k^{\alpha_{1}}+1\right)}{2}=\frac{3+c}{2} k^{2 \alpha_{1}}+\frac{9+c}{2} k^{\alpha_{1}} .
\end{aligned}
$$

Подсчитаем объем ИГ $U_{q}$ при $2 \leqslant q \leqslant M$.

ИГ $U_{q}$ содержит в себе ИГ $U_{q-1}$.

Из каждой вершины $\vartheta_{i}^{q-1}, i=2, \ldots, k^{\alpha_{1}+\ldots+\alpha_{q-1}}$, исходит ИГ $U_{\mathcal{N}_{i-1}^{q}, l_{i-1}^{q-1}, l_{i}^{4-1}}^{1}$, объем которого согласно (16) равен $(2+c)\left(k^{\alpha_{q}}-1\right)+1$.

Из каждой вершины $\beta_{i}^{q-1}, i=1, \ldots, k^{\alpha_{1}+\ldots+\alpha_{q-1}}$, исходит ИГ $U_{\mathcal{N}_{i}^{q}, l_{i}^{q-1}, l_{i+1}^{q-1}}^{2}$, объем которого согласно $(17)$ равен $(2+c)\left(k^{\alpha_{4}}-1\right)+1$.

Из каждой вершины $\gamma_{i}^{q-1}, i=2, \ldots, k^{\alpha_{1}+\ldots+\alpha_{q-1}}+1$, исходит ИГ $U_{\mathcal{N}_{i-1}^{q}, l_{i-1}^{q-1}, l_{i}^{q-1}}^{1}$. Его объем равен $(2+c)\left(k^{\alpha_{q}}-1\right)+1$.

Из каждой вершины $\theta_{q i j}, j=1, \ldots, k^{\alpha_{q}}-1$, исходит по 2 ребра. Из каждой вершины $\theta_{q i j}^{\prime \prime}, j=1, \ldots, k^{\alpha_{q}}-2$, исходит ИГ $U_{Z_{i-1}^{q, j}, l_{(i-2) k}^{q} \alpha_{q+j+1}, l_{i}^{q-1}}^{2}$, объем которого равен $(2+c)\left(k^{\alpha_{q}}-j-1\right)+1$. Таким образом, объем ИГ, растущего из вершины $\gamma_{i}^{q-1}$, равен

$$
\begin{aligned}
(2+c)\left(k^{\alpha_{\varphi}}-1\right)+1+2\left(k^{\alpha_{\varphi}}-1\right)+\sum_{j=1}^{k^{\alpha_{q}-2}}\left((2+c)\left(k^{\alpha_{q}}-j-1\right)+1\right) & =\frac{2+c}{2} k^{2 \alpha_{\varphi}}+\frac{4-c}{2} k^{\alpha_{q}}-3 .
\end{aligned}
$$

Если $q<M$, то в вершины $\vartheta_{t}^{q}, t=2, \ldots, k^{\alpha_{1}+\ldots+\alpha_{q}}$, из каждой вершины

$$
\theta_{q i j}^{\prime \prime}, \quad i=2, \ldots, k^{\alpha_{1}+\ldots+\alpha_{q-1}}+1, \quad j=1, \ldots, k^{\alpha_{q}}-1
$$

и каждой вершины $\tau_{q i j}^{\prime}, i=2, \ldots, k^{\alpha_{1}+\ldots+\alpha_{q-1}}, j=1, \ldots, k^{\alpha_{q}}$, ведет по одному ребру. Следовательно, суммарное число ребер, ведущих в вершины $\vartheta_{t}^{q}$, равно

$$
k^{\alpha_{1}+\ldots+\alpha_{q-1}}\left(k^{\alpha_{q}}-1\right)+\left(k^{\alpha_{1}+\ldots+\alpha_{q-1}}-1\right) k^{\alpha_{q}}=2 k^{\alpha_{1}+\ldots+\alpha_{q}}-k^{\alpha_{1}+\ldots+\alpha_{q-1}}-k^{\alpha_{q}} .
$$

В вершины $\beta_{s}^{q}, s=1, \ldots, k^{\alpha_{1}+\ldots+\alpha_{q}}$, из каждой вершины

$$
\mu_{i j t}^{q}, \quad i=2, \ldots, k^{\alpha_{1}+\ldots+\alpha_{q-1}}+1, \quad j=1, \ldots, k^{\alpha_{q}}-1, \quad t=j+1, \ldots, k^{\alpha_{q}},
$$

и каждой вершины $\tau_{q i j}^{\prime \prime}, i=1, \ldots, k^{\alpha_{1}+\ldots+\alpha_{q-1}}, j=1, \ldots, k^{\alpha_{q}}$, ведет по одному ребру. Следовательно, суммарное число ребер, ведущих в вершины $\beta_{s}^{q}$, равно

$$
k^{\alpha_{1}+\ldots+\alpha_{q-1}} \sum_{j=1}^{k^{\alpha_{q}}-1}\left(k^{\alpha_{q}}-j\right)+k^{\alpha_{1}+\ldots+\alpha_{q-1}} k^{\alpha_{q}}=k^{\alpha_{1}+\ldots+\alpha_{q}} \frac{k^{\alpha_{q}}+1}{2} .
$$

Тем самым при $2 \leqslant q<M$

$$
\begin{aligned}
Q\left(U_{q}\right)=Q & \left(U_{q-1}\right)+\left((2+c)\left(k^{\alpha_{u}}-1\right)+1\right)\left(k^{\alpha_{1}+\ldots+\alpha_{q-1}}-1\right) \\
& +\left((2+c)\left(k^{\alpha_{u}}-1\right)+1\right) k^{\alpha_{1}+\ldots+\alpha_{q-1}} \\
& +\left((2+c) k^{2 \alpha_{u}} / 2+(4-c) k^{\alpha_{u}} / 2-3\right) k^{\alpha_{1}+\ldots+\alpha_{q-1}} \\
& +2 k^{\alpha_{1}+\ldots+\alpha_{q}}-k^{\alpha_{1}+\ldots+\alpha_{q-1}}-k^{\alpha_{q}}+k^{\alpha_{1}+\ldots+\alpha_{u}}\left(k^{\alpha_{q}}+1\right) / 2 \\
=U & \left(Q_{q-1}\right)+(3+c) k^{\alpha_{1}+\ldots+\alpha_{q-1}+2 \alpha_{q}} / 2+\mathcal{O}\left(k^{\alpha_{1}+\ldots+\alpha_{q}}\right) .
\end{aligned}
$$


Учитывая, что суммарное число ребер, ведущих в вершины $\vartheta_{t}^{q}$, равно $\sigma\left(k^{\alpha_{1}+\ldots+\alpha_{q}}\right)$, и есть еще другие слагаемые, имеющие тот же порядок, получаем, что

$$
Q\left(U_{M}\right)=\frac{3+c}{2} \sum_{q=1}^{M} k^{\alpha_{1}+\ldots+\alpha_{q-1}+2 \alpha_{q}}+\odot(k) .
$$

Вычислим объем информационного графа $U^{*}$.

Из (33) легко видеть, что

$$
\left|A_{s, i, j}^{q}\right|=(j-i) k^{1-\left(\alpha_{1}+\ldots+\alpha_{q}\right)} .
$$

Из каждой вершины

$\delta_{s i j}^{q}, \quad q=1, \ldots, M, \quad s=1, \ldots, k^{\alpha_{1}+\ldots+\alpha_{q-1}}, \quad i=2, \ldots, k^{\alpha_{q}}-1, \quad j=i+1, \ldots, k^{\alpha_{q}}$

(при $q=1$ индексы меняются так: $s=1, i=1, \ldots, k^{\alpha_{1}}-1, j=i+1, \ldots, k^{\alpha_{1}}$ ) исходит ИГ $U_{A_{s, i, j}^{q}}^{3}$, объем которого согласно (18) и (35) равен

$$
Q\left(U_{A_{s, i, j}^{q}}^{3}\right) \leqslant 4(j-i) k^{1-\left(\alpha_{1}+\ldots+\alpha_{q}\right)}-1+6 c\left[\log _{2}\left((j-i) k^{1-\left(\alpha_{1}+\ldots+\alpha_{q}\right)}\right)\right] .
$$

Суммируя по всем $q, s, i, j$, получаем, что объем ИГ, растущих из вершин $\delta_{s i j}^{q}$, (обозначим его $Q_{1}$ ) не превышает

$$
\begin{aligned}
& Q_{1}<\sum_{q=1}^{M} \sum_{s=1}^{k^{\alpha_{1}}+\ldots+\alpha_{q-1}} \sum_{i=2}^{k^{\alpha_{q}}-1} \sum_{j=i+1}^{k^{\alpha_{q}}} 4(j-i) k^{1-\left(\alpha_{1}+\ldots+\alpha_{q}\right)} \\
& +\sum_{q=1}^{M} \sum_{s=1}^{k^{\alpha_{1}+\ldots+\alpha_{q}-1}} \sum_{i=2}^{k^{\alpha_{q}}-1} \sum_{j=i+1}^{k^{\alpha_{q}}} 6 c\left[\log _{2}\left((j-i) k^{1-\left(\alpha_{1}+\ldots+\alpha_{q}\right)}\right)\right] \\
& +\sum_{j=2}^{k^{\alpha_{1}}}\left(4(j-1) k^{1-\alpha_{1}}+6 c\left[\log _{2}\left((j-1) k^{1-\alpha_{1}}\right)\right]\right) \text {. }
\end{aligned}
$$

Оценим первое слагаемое, которое обозначим $Q_{11}$ :

$$
\begin{aligned}
Q_{11} & =\sum_{q=1}^{M} \sum_{s=1}^{k^{\alpha_{1}+\ldots+\alpha_{q-1}}} \sum_{i=2}^{k^{\alpha_{q}}-1} \sum_{j=i+1}^{k^{\alpha_{q}}} 4(j-i) k^{1-\left(\alpha_{1}+\ldots+\alpha_{q}\right)} \\
& =2 \sum_{q=1}^{M} k^{1-\alpha_{q}} \sum_{i=2}^{k^{\alpha_{q}-1}}\left(k^{\alpha_{q}}-i\right)\left(k^{\alpha_{q}}-i+1\right)=2 \sum_{q=1}^{M} k^{1-\alpha_{q}} \sum_{i=1}^{k^{\alpha_{q}-2}} i(i+1) \\
& =2 \sum_{q=1}^{M} k^{1-\alpha_{q}}\left(\frac{\left(k^{\alpha_{q}}-2\right)\left(k^{\alpha_{q}}-1\right)\left(2 k^{\alpha_{q}}-4+1\right)}{6}+\frac{\left(k^{\alpha_{q}}-2\right)\left(k^{\alpha_{q}}-1\right)}{2}\right) \\
& =\frac{2}{3} \sum_{q=1}^{M} k\left(k^{\alpha_{q}}-2\right)\left(k^{\alpha_{q}}-1\right)=\frac{2}{3} \sum_{q=1}^{M} k^{1+2 \alpha_{q}}+O\left(\sum_{q=1}^{M} k^{1+\alpha_{q}}\right) .
\end{aligned}
$$


Оценим второе слагаемое, которое обозначим $Q_{12}$ :

$$
\begin{aligned}
Q_{12} & =\sum_{q=1}^{M} \sum_{s=1}^{k^{\alpha_{1}+\ldots+\alpha_{q-1}}} \sum_{i=2}^{k^{\alpha_{q}}-1} \sum_{j=i+1}^{k^{\alpha_{q}}} 6 c\left[\log _{2}\left((j-i) k^{1-\left(\alpha_{1}+\ldots+\alpha_{q}\right)}\right)\right] \\
& <6 c \sum_{q=1}^{M} k^{\alpha_{1}+\ldots+\alpha_{q-1}} \sum_{i=2}^{k^{\alpha_{q}}-1} \sum_{j=i+1}^{k^{\alpha_{q}}} \log _{2}\left(k^{1-\left(\alpha_{1}+\ldots+\alpha_{q-1}\right)}\right) \\
& =3 c \log _{2} k \sum_{q=1}^{M} k^{\alpha_{1}+\ldots+\alpha_{q-1}}\left(1-\left(\alpha_{1}+\ldots+\alpha_{q-1}\right)\right)\left(k^{\alpha_{q}}-2\right)\left(k^{\alpha_{q}}-1\right) . \\
& <3 c \log _{2} k \sum_{q=1}^{M} k^{\alpha_{1}+\ldots+\alpha_{q-1}+2 \alpha_{q}}\left(1-\left(\alpha_{1}+\ldots+\alpha_{q-1}\right)\right) .
\end{aligned}
$$

Из каждой вершины

$$
\eta_{i j}^{q}, \quad q=2, \ldots, M, \quad i=2, \ldots, k^{\alpha_{1}+\ldots+\alpha_{q-1}}, \quad j=1, \ldots, k^{\alpha_{4}}-1,
$$

исходит ИГ $U_{A_{i-1, j+1, k^{q}+1}^{3}}^{3}$, объем которого согласно (18) и (35) равен

$$
Q\left(U_{A_{i-1, j+1, k^{\alpha}+1}^{3}}^{3}\right) \leqslant 4\left(k^{\alpha_{q}}-j\right) k^{1-\left(\alpha_{1}+\ldots+\alpha_{q}\right)}-1+6 c\left[\log _{2}\left(\left(k^{\alpha_{q}}-j\right) k^{1-\left(\alpha_{1}+\ldots+\alpha_{q}\right)}\right)\right] .
$$

Суммируя по всем $q, i, j$, получаем, что объем ИГ, растущих из вершин $\eta_{i j}^{q}$ (обозначим его $Q_{2}$ ), не превышает

$$
\begin{aligned}
Q_{2} & <\sum_{q=2}^{M} \sum_{i=1}^{k^{\alpha_{1}+\ldots+\alpha_{q-1}}} \sum_{j=1}^{k^{\alpha_{q}}-1}(4+6 c)\left(k^{\alpha_{q}}-j\right) k^{1-\left(\alpha_{1}+\ldots+\alpha_{q}\right)} \\
& =(4+6 c) \sum_{q=2}^{M} k^{1-\alpha_{q}} \sum_{j=1}^{k^{\alpha_{q}-1}} j<(2+3 c) \sum_{q=2}^{M} k^{1+\alpha_{q}}
\end{aligned}
$$

Из каждой вершины $\zeta_{i j}^{q}, q=2, \ldots, M, i=1, \ldots, k^{\alpha_{1}+\ldots+\alpha_{q-1}}, j=2, \ldots, k^{\alpha_{q}}$, исходит ИГ $U_{A_{i, 1, j}^{4}}^{3}$, объем которого согласно (18) и (35) равен

$$
Q\left(U_{A_{i, 1, j}^{q}}^{3}\right) \leqslant 4(j-1) k^{1-\left(\alpha_{1}+\ldots+\alpha_{q}\right)}-1+6 c\left(\log _{2}\left((j-1) k^{1-\left(\alpha_{1}+\ldots+\alpha_{q}\right)}\right)\right) .
$$

Суммируя по всем $q, i, j$, получаем, что объем ИГ, растущих из вершин $\zeta_{i j}^{q}$ (обозначим его $Q_{3}$ ), удовлетворяет неравенствам

$$
\begin{aligned}
Q_{3} & <\sum_{q=2}^{M} \sum_{i=1}^{k^{\alpha_{1}+\ldots+\alpha_{q-1}}} \sum_{j=2}^{k^{\alpha_{q}}}(4+6 c)(j-1) k^{1-\left(\alpha_{1}+\ldots+\alpha_{q}\right)} \\
& =(4+6 c) \sum_{q=2}^{M} k^{1-\alpha_{q}} \sum_{j=1}^{k^{\alpha_{q}-1}} j<(2+3 c) \sum_{q=2}^{M} k^{1+\alpha_{q}}
\end{aligned}
$$

Из каждой вершины $\beta_{j}^{M}, j=1, \ldots, k^{\alpha_{1}+\ldots+\alpha_{M}}$, исходит по одному ребру, следовательно, суммарное число ребер, исходящих из вершин $\beta_{j}^{M}$, равно

$$
Q_{4}=k^{\alpha_{1}+\ldots+\alpha_{M}}=k .
$$


Исходя из (37), положим

$$
\alpha_{1}=\alpha_{2}=\ldots=\alpha_{M}=1 / M \text {. }
$$

Согласно (34) и (42)

$$
\begin{aligned}
Q\left(U_{M}\right) & =\frac{3+c}{2} \sum_{q=1}^{M} k^{(q+1) / M}+\mathcal{O}(k)=\frac{3+c}{2} k^{1+1 / M} \sum_{q=1}^{M} k^{-q / M}+\mathcal{O}(k) \\
& =\frac{3+c}{2} k^{1+1 / M} \frac{1-k^{-1}}{1-k^{-1 / M}}+\mathcal{O}(k)=\mathcal{O}\left(k^{1+1 / M}\right) .
\end{aligned}
$$

Поскольку

$$
\begin{aligned}
\sum_{i=0}^{n} d^{i}(i+1) & =\sum_{i=0}^{n} \sum_{j=i}^{n} d^{j} \\
& =\sum_{i=0}^{n} d^{i} \frac{1-d^{n-i+1}}{1-d}=\frac{1}{1-d} \sum_{i=0}^{n}\left(d^{i}-d^{n+1}\right) \\
& =\frac{1}{(1-d)^{2}}\left(1-(n+2) d^{n+1}+(n+1) d^{n+2}\right)
\end{aligned}
$$

при $\alpha_{1}=\alpha_{2}=\ldots=\alpha_{q}=1 / M$ согласно (38)

$$
\begin{aligned}
Q_{12} & <3 c \log _{2} k \sum_{q=1}^{M} k^{(q+1) / M}\left(1-\frac{q-1}{m}\right) \\
& =\frac{3 c}{M} k^{1+1 / M} \log _{2} k \sum_{q=1}^{M} k^{(q-M) / M}(M-q+1) \\
& =\frac{3 c}{M} k^{1+1 / M} \log _{2} k \sum_{q=0}^{M-1} k^{-q / M}(q+1) \\
& =\frac{3 c}{M} k^{1+1 / M} \frac{\log _{2} k}{\left(1-k^{-1 / M}\right)^{2}}\left(1-(M+1) k^{-1}+M k^{-M-1 / M}\right) \\
& =\mathcal{O}\left(\frac{1}{M} k^{1+1 / M} \ln k\right) .
\end{aligned}
$$

Таким образом, согласно (43), (36), (37), (44), (39), (40), (41) получим, что

$$
\begin{aligned}
Q(U) & =Q\left(U_{M}\right)+Q_{1}+Q_{2}+Q_{3}+Q_{4} \\
& \leqslant \frac{2}{3} M k^{1+2 / M}+\odot\left(M k^{1+1 / M}+\frac{\ln k}{M} k^{1+1 / M}\right) .
\end{aligned}
$$

\section{7. Сложность информационного графа}

Если $X$ - множество запросов с заданным на нем вероятностным пространством $\langle X, \sigma, \mathbf{P}\rangle, \mathscr{F}-$ базовое множество функций, определенных на $X, U-$ ИГ над $\mathscr{F}, \beta-$ вершина ИГ $U$, то сложностью вершины $\beta$ называется число $\mathbf{P}\left(N_{\varphi_{\beta}}\right)$, сложностью 
предикатного ребра $(\beta, \alpha)$ называется число $\mathbf{P}\left(N_{\varphi_{\beta}}\right)$, то есть сложность начала ребра, сложностью переключательного ребра $(\beta, \alpha)$ называется число $\mathbf{P}\left(N_{\varphi_{\beta}}\right) / \psi_{\beta}$, где, напомним, $\psi_{\beta}-$ количество ребер, исходящих из вершины $\beta$.

Как показано в [27], сложность ИГ равна сумме сложностей ребер ИГ.

Прежде чем вычислять сложность ИГ $U^{*}$, докажем одно вспомогательное утверждение.

Пусть $\beta$ - вершина некоторого ИГ $U$. Скажем что подграф $U^{\prime}$ графа $U$ является зависимым от $\beta$, если он содержит вершину $\beta$, является связным, состоит только из таких вершин $\alpha$ ИГ $U$, что любая цепь, ведущая из корня ИГ $U$ в вершину $\alpha$, проходит через вершину $\beta$ и содержит все ребра ИГ $U$, исходящие из вершин $U^{\prime}$.

Так, например, если вершина $\beta$ является корнем связного ИГ $U$, то ИГ $U$ будет зависимым от $\beta$. А для произвольной вершицы $\beta$ любой зависимый от $\beta$ граф, по крайней мере, содержит все ребра, исходящие из вершины $\beta$.

Договоримся через $\mathrm{P}(A \mid B)$ обозначать условную вероятность события $A$ при условии осуществления события $B$.

Пусть $X$ - множество запросов с заданным на нем вероятностным пространством $\langle X, \sigma, \mathbf{P}\rangle$. Пусть $X^{\prime} \in \sigma$. Вероятностным пространством, порожденным множеством $X^{\prime}$, назовем вероятностное пространство $\left\langle X^{\prime}, \sigma^{\prime}, \mathbf{P}^{\prime}\right\rangle$, где $\sigma^{\prime}=\left\{B \in \sigma: B \subseteq X^{\prime}\right\}$, $\mathbf{P}^{\prime}$ - такая вероятностная мера на $\sigma^{\prime}$, что $\mathbf{P}^{\prime}(B)=\mathbf{P}\left(B \mid X^{\prime}\right)$ для любого $B \in \sigma^{\prime}$.

Лемма 1. Пусть $\beta$ - вериина некоторого ИГ $U$ над базовым множеством, функции которого определенъ на множестве запросов $X,\langle X, \sigma, \mathbf{P}\rangle$ - вероятностное пространство над $X, X^{\prime}=N_{\varphi_{\beta}},\left\langle X^{\prime}, \sigma^{\prime}, \mathbf{P}^{\prime}\right\rangle$ - вероятностное пространство, порожденное множеством $X^{\prime}, U^{\prime}-$ зависимый от $\beta$ подграф ИГ $U, T^{\prime}-$ сложность ИГ $U^{\prime}$ (ках отделъного ИГ с корнем в вершине $\beta$ ) для вероятностного пространства $\left\langle X^{\prime}, \sigma^{\prime}, \mathbf{P}^{\prime}\right\rangle$. Тогда сложность $U^{\prime}$ как подграфа ИГ $U$ для вероятностного пространства $\langle X, \sigma, \mathbf{P}\rangle$ равна $T\left(U^{\prime}\right)=\mathbf{P}\left(X^{\prime}\right) T^{\prime}$.

Доказательство. Рассмотрим произвольную вершину $\alpha$ подграфа $U^{\prime}$. Из определения зависимого подграфа следует, что $N_{\varphi_{\alpha}} \subseteq N_{\varphi_{\beta}}=X^{\prime}$. Следовательно,

$$
\mathbf{P}\left(N_{\varphi_{\alpha}}\right)=\mathbf{P}\left(N_{\varphi_{\alpha}} \cap X^{\prime}\right)=\mathbf{P}\left(X^{\prime}\right) \mathbf{P}\left(N_{\varphi_{\alpha}} \mid X^{\prime}\right)=\mathbf{P}\left(X^{\prime}\right) \mathbf{P}^{\prime}\left(N_{\varphi_{\alpha}}\right) .
$$

Отсюда сразу следует утверждение леммы.

Вычислим сложность ИГ $U^{*}$.

Пусть $\beta$ - некоторая вершина ИГ $U^{*}$. Чтобы сократить этажность формул, договоримся обозначать $N(\beta)=N_{\varphi_{\beta}}$.

Введем следующие множества вершин ИГ $U^{*}$ :

$$
\begin{aligned}
& \mathscr{B}_{1}=\left\{\theta_{i}: i=1,2, \ldots, k^{1 / M}\right\} \\
& \mathscr{B}_{2}=\left\{\theta_{i}^{\prime \prime}: i=1,2, \ldots, k^{1 / M}\right\} \\
& \mathscr{B}_{3}=\left\{\theta_{i j}: i=1,2, \ldots, k^{1 / M}-1, j=i+1, \ldots, k^{1 / M}\right\}, \\
& \mathscr{B}_{4}^{q}=\left\{\theta_{q i j}: i=2, \ldots, k^{(q-1) / M}+1, j=1, \ldots, k^{1 / M}-1\right\}, \quad q=2, \ldots, M, \\
& \mathscr{B}^{4}=\bigcup_{q=2}^{M} \mathscr{B}_{4}^{q} .
\end{aligned}
$$


Обозначим через $T_{q}, q=1,2, \ldots, M$, суммарную сложность ребер ИГ $U_{q}$ за исключением ребер, исходящих из вершин множества $\mathscr{B}_{4}$. Через $\widehat{T}_{q}(\tilde{x}), q=1,2, \ldots, M$, обозначим сложность ИГ $U_{q}$ на запросе $\tilde{x}$ минус количество вычисленных переключателей, приписанных вершинам из множества $\mathscr{B}_{4}$.

Покажем по индукции, что

$$
T_{q} \leqslant 6 q, \quad \widehat{T}_{q}(\tilde{x}) \leqslant 6 q+\frac{2 q}{M} \log _{2} k
$$

для любого $\tilde{x} \in X_{\text {int 2 }}$.

Пусть $q=1$. Из соотношений (15), (26), (27) легко получаем следующие утверждения:

$$
\forall \beta_{1}, \beta_{2} \in \mathscr{B}_{i}\left(\beta_{1} \neq \beta_{2} \Rightarrow N\left(\beta_{1}\right) \cap N\left(\beta_{2}\right)=\varnothing\right), \quad i=1,2,3
$$

Из корня ИГ $U^{*}$ растет ИГ $U_{\mathcal{N}^{1}, 0,1}^{1}$, сложность которого оценивается соотношением (16), вершинам $\theta_{i}$ приписаны переключатели $g_{l_{i}^{1}}^{4}$, из вершин $\theta_{i}^{\prime \prime}$ исходят ИГ $U_{Z^{1, i}, l_{i}^{1}, 1}^{2}$, зависимые от $\theta_{i}^{\prime \prime}$ и сложность которых оценивается соотношением (17), а из вершин $\theta_{i j}$ исходят ребра с предикатом тождественная 1 , поэтому с учетом (45) согласно лемме 1

$$
T_{1}=2+\sum_{\beta \in \mathscr{B}_{1}} \mathbf{P}(N(\beta))+\sum_{\beta \in \mathscr{B}_{2}} 2 \mathbf{P}(N(\beta))+\sum_{\beta \in \mathscr{B}_{3}} \mathbf{P}(N(\beta)) \leqslant 6,
$$

и для любого $\tilde{x} \in X_{\text {int } 2}$

$$
\left.\widehat{T}_{1}(\tilde{x}) \leqslant 1+\right] \log _{2}\left(k^{1 / M}+1\right)[+1+1+] \log _{2}\left(k^{1 / M}+1\right)\left[+1 \leqslant 6+\frac{2}{M} \log _{2} k\right.
$$

Рассмотрим индуктивный переход. Пусть $q \in\{2, \ldots, M\}$ и утверждение индукции выполняется для $q-1$. Введем обозначения

$$
\begin{aligned}
\mathscr{B}_{5}^{q}= & \left\{\vartheta_{i}^{q-1}: i=2, \ldots, k^{(q-1) / M}\right\} \cup\left\{\gamma_{i}^{q-1}: i=2, \ldots, k^{(q-1) / M}+1\right\}, \\
\mathscr{B}_{6}^{q}= & \left\{\beta_{i}^{q-1}: i=1, \ldots, k^{(q-1) / M}\right\} \\
& \cup\left\{\theta_{q i j}^{\prime \prime}: i=2, \ldots, k^{(q-1) / M}+1, j=1, \ldots, k^{1 / M}-1\right\}, \\
\mathscr{B}_{7}^{q}= & \left\{\tau_{q i j}^{\prime}: i=2, \ldots, k^{(q-1) / M}, j=1, \ldots, k^{1 / M}\right\} \\
& \cup\left\{\theta_{q i j}^{\prime \prime}: i=2, \ldots, k^{(q-1) / M}+1, j=1, \ldots, k^{1 / M}-1\right\}, \\
\mathscr{B}_{8}^{q}= & \left\{\tau_{q i j}^{\prime \prime}: i=1, \ldots, k^{(q-1) / M}, j=1, \ldots, k^{1 / M}\right\} \\
& \cup\left\{\mu_{i j t}^{q}: i=2, \ldots, k^{(q-1) / M}+1, j=1, \ldots, k^{1 / M}-1, t=j+1, \ldots, k^{1 / M}\right\} .
\end{aligned}
$$

Из соотношений $(20),(21),(22),(28),(29),(31),(32)$ легко получаем следующие утверждения:

$$
\forall \beta_{1}, \beta_{2} \in \mathscr{B}_{i}^{q}\left(\beta_{1} \neq \beta_{2} \Rightarrow N\left(\beta_{1}\right) \cap N\left(\beta_{2}\right)=\varnothing\right), \quad i=5,6,7,8 .
$$

Из вершин $\vartheta_{i}^{q-1}$ и $\gamma_{i}^{q-1}$ исходят зависимые от этих вершин ИГ $U_{\mathcal{N}_{i-1}^{q}, l_{i-1}^{q-1}, l_{i}^{y-1}}^{1}$, сложность которых оценивается соотношением (16), из вершин $\beta_{i}^{q-1}$ исходят ИГ 


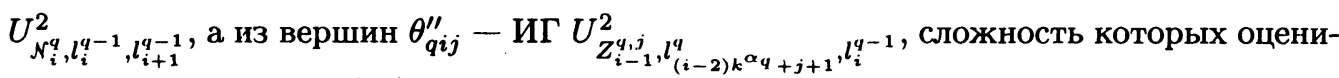
вается соотношением (17) и которые зависимы от своих корневых вершин, из каждой вершины из множества $\mathscr{B}_{7}^{q}$ исходит ребро с предикатом тождественная 1 , ведущее в вершины $\vartheta_{i}^{q}$, а из каждой вершины из множества $\mathscr{B}_{8}^{q}$ исходит ребро с предикатом тождественная 1 , ведущее в вершины $\beta_{i}^{q}$, поэтому с учетом (46) согласно лемме 1

$$
\begin{aligned}
T_{q}=T_{q-1}+\sum_{\beta \in \mathscr{B}_{5}^{q}} 2 \mathbf{P}(N(\beta))+\sum_{\beta \in \mathscr{B}_{6}^{q}} 2 \mathbf{P}(N(\beta)) & +\sum_{\beta \in \mathscr{B}_{7}^{q}} \mathbf{P}(N(\beta)) \\
& +\sum_{\beta \in \mathscr{B}_{8}^{q}} \mathbf{P}(N(\beta)) \leqslant 6(q-1)+6=6 q,
\end{aligned}
$$

и для любого $\tilde{x} \in X_{\text {int } 2}$

$$
\begin{aligned}
\widehat{T}_{q}(\tilde{x}) & \left.\leqslant \widehat{T}_{q-1}(\tilde{x})+1+\right] \log _{2}\left(k^{1 / M}+1\right)[+1+] \log _{2}\left(k^{1 / M}+1\right)[+1+1 \\
& \leqslant 6 q+\frac{2 q}{M} \log _{2} k .
\end{aligned}
$$

При $q=M$ нам надо делать на одно вычисление меньше, так как в ИГ $U_{M}$ нет вершин типа $\vartheta_{i}^{M}$, то есть

$$
T_{M} \leqslant 6 M-1, \quad \widehat{T}_{M}(\tilde{x}) \leqslant 6 M-1+2 \log _{2} k
$$

для любого $\tilde{x} \in X_{\text {int 2 }}$.

Введем обозначения

$$
\begin{aligned}
\mathscr{B}_{9}^{1}= & \left\{\delta_{1 i j}^{1}: i=1, \ldots, k^{1 / M}-1, j=i+1, \ldots, k^{1 / M}\right\}, \\
\mathscr{B}_{9}^{q}= & \left\{\delta_{s i j}^{q}: s=1, \ldots, k^{(q-1) / M}, i=2, \ldots, k^{1 / M}-1, j=i+1, \ldots, k^{1 / M}\right\}, \\
& q=2, \ldots, M, \\
\mathscr{B}_{10}^{q}= & \left\{\eta_{i j}^{q}: i=2, \ldots, k^{(q-1) / M}, j=1, \ldots, k^{1 / M}-1\right\}, \quad q=2, \ldots, M, \\
\mathscr{B}_{11}^{q}= & \left\{\zeta_{i j}^{q}: i=1, \ldots, k^{(q-1) / M}, j=2, \ldots, k^{1 / M}-1\right\}, \quad q=2, \ldots, M, \\
\mathscr{B}_{9}= & \bigcup_{q=1}^{M} \mathscr{B}_{9}^{q}, \\
\mathscr{B}_{10}= & \bigcup_{q=2}^{M} \mathscr{B}_{10}^{q}, \\
\mathscr{B}_{11}= & \bigcup_{q=2}^{M} \mathscr{B}_{11}^{q}, \\
\mathscr{B}_{12}= & \left\{\beta_{i}^{M}: i=1, \ldots, k\right\}, \\
\mathscr{B}_{13}= & \mathscr{B}_{9} \cup \mathscr{B}_{10} \cup \mathscr{B}_{11} .
\end{aligned}
$$

С помощью (21), (23), (24), (25), (30) непосредственно проверяется справедливость следующих утверждений:

$$
\forall \beta_{1}, \beta_{2} \in \mathscr{B}_{9} \cup \mathscr{B}_{12}\left(\beta_{1} \neq \beta_{2} \Rightarrow N\left(\beta_{1}\right) \cap N\left(\beta_{2}\right)=\varnothing\right),
$$

$\forall q \in\{2, \ldots, M\}\left(\forall \beta_{1}, \beta_{2} \in \mathscr{B}_{i}^{q} \cup \mathscr{B}_{4}^{q}\left(\beta_{1} \neq \beta_{2} \Rightarrow N\left(\beta_{1}\right) \cap N\left(\beta_{2}\right)=\varnothing\right)\right), i=10,11$, (48)

$$
\forall \beta_{1}, \beta_{2} \in \mathscr{B}_{13}\left(\beta_{1} \neq \beta_{2} \Rightarrow\left(N\left(\beta_{1}\right) \cap N\left(\beta_{2}\right)=\varnothing \vee A_{\beta_{1}} \cap A_{\beta_{2}}=\varnothing\right)\right) \text {. }
$$


Пусть $\beta \in \mathscr{B}_{13}$, тогда для некоторых $q, s, i, j$ из вершины $\beta$ исходит ИГ $U_{A_{s i j}^{q}}^{3}$, зависимый от вершины $\beta$. Положим

$$
A_{\beta}=\left\{\left(y_{p}^{1}, y_{p}^{2}\right) \in V: l_{(s-1) k^{\alpha_{q}+i}}^{q} \leqslant y_{p}^{1}<l_{(s-1) k^{\alpha_{q}+j}}^{q}\right\}
$$

Согласно лемме 1 и (18) для данной вершины $\beta$

$$
\begin{aligned}
T\left(U_{A_{s i j}^{q}}^{3}\right) & =\mathbf{P}(N(\beta))\left(5+\sum_{y \in A_{s i j}^{q}} \mathbf{P}^{\prime}\left(O\left(y, \rho_{\mathrm{int}}\right)\right)\right) \\
& =5 \mathbf{P}(N(\beta))+\sum_{\tilde{y} \in A_{\beta}} \mathbf{P}\left(O\left(\tilde{y}, \rho_{\mathrm{int} 2}\right) \cap N(\beta)\right) .
\end{aligned}
$$

Учитывая, что при попадании в вершину из $\mathscr{B}_{12}$ вычисляется один предикат, приписанный ребру, исходящему из этой вершины, а при попадании в вершину из $\mathscr{B}_{4}$ вычисляется один переключатель, приписанный этой вершине, находим, что

$$
\begin{aligned}
& T\left(U^{*}\right) \leqslant T_{M}+\sum_{\beta \in \mathscr{B}_{4}} \mathbf{P}(N(\beta))+\sum_{\beta \in \mathscr{P}_{12}} \mathbf{P}(N(\beta)) \\
& +\sum_{\beta \in \mathscr{B}_{13}}\left(5 \mathbf{P}(N(\beta))+\sum_{\tilde{y} \in A_{\beta}} \mathbf{P}\left(O\left(\tilde{y}, \rho_{\text {int } 2}\right) \cap N(\beta)\right)\right) \\
& \leqslant T_{M}+5 \sum_{\beta \in \mathscr{B}_{9} \cup \mathscr{B}_{12}} \mathbf{P}(N(\beta))+5 \sum_{q=2}^{M} \sum_{\beta \in \mathscr{B}_{4}^{q} \cup \mathscr{B}_{10}^{q}} \mathbf{P}(N(\beta)) \\
& +5 \sum_{q=2}^{M} \sum_{\beta \in \mathscr{B}_{11}^{q}} \mathbf{P}(N(\beta))+\sum_{\tilde{y} \in V} \sum_{\beta \in \mathscr{B}_{13}: \tilde{y} \in A_{\beta} .} \mathbf{P}\left(O\left(\tilde{y}, \rho_{\text {int } 2}\right) \cap N(\beta)\right) \\
& \leqslant 16 M-6+\sum_{\tilde{y} \in V} \mathbf{P}\left(O\left(\tilde{y}, \rho_{\text {int } 2}\right)\right) \text {. }
\end{aligned}
$$

Согласно (47) для любого запроса мы можем попасть не более чем в одну вершину из $\mathscr{B} 9 \cup \mathscr{B}_{12}$, следовательно, при этом мы в худшем случае выполним $2+\log _{2} k$ вычислений плюс перечисление ответа. Согласно (48) для каждого $q=2, \ldots, M$ и любого запроса мы можем попасть не более чем в одну вершину из $\mathscr{B}_{4} \cup \mathscr{B}_{10}$ и не более чем в одну вершину из $\Re_{11}$ и в каждом из этих случаев мы выполним не более чем $2+(1 / M) \log _{2} k$ вычислений плюс перечисление ответа. Тем самым для любого запроса $\tilde{x} \in X_{\text {int } 2}$

$$
\begin{aligned}
T\left(U^{*}, x\right) & \leqslant \widehat{T}_{M}+2+\log _{2} k+4(M-1)+\frac{M-1}{m} \log _{2} k\left|\left\{\tilde{y} \in V: \tilde{x} \rho_{\text {int } 2} \tilde{y}\right\}\right| \\
& <10 M+4 \log _{2} k+\left|\left\{\tilde{y} \in V: \tilde{x} \rho_{\text {int } 2} \tilde{y}\right\}\right| .
\end{aligned}
$$

Тем самым теорема 1 полностью доказана. 


\section{Список литературы}

1. Ли Д., Препарата Ф., Вычислительная геометрия. Обзор. Киберн. сб. (1987) 24, 5-96.

2. Кнут Д., Искусство программироваяия для ЭВМ. Сортировка и поиск, т. 3. Мир, Москва, 1978.

3. Loftsgaarden D. O., Queensberry C. P., A nonparametric density function. Ann. Math. Stat. (1965) 36, 1049-1051.

4. Lauter U., 4-dimensional binary search trees as a means to speed up associative searches in design verification of integrated circuits. J. Design Automation and Fault Tolerant Computing (1978) 2, 241-247.

5. Гасанов Э. Э., Мгновенно решаемые задачи поиска. Дискретная математиха (1996) 8, № 3, 119-134.

6. Препарата Ф., Шеймос М., Вычислительная геометрия. Вөедение. Мир, Москва, 1989.

7. Bentley J. L., Multidimensional binary search trees used for associative searching. Commun. Ass. Comput. Mach. (1975) 18, 509-517.

8. Bentley J. L., Decomposable searching problems. Inform. Processing Letters (1979) 8, 244251.

9. Bentley J. L., Friedman J. H., Data structures for range searching. Comput. Surveys (1979) 11, 397-409.

10. Bentley J. L., Maurer H. A., Efficient worst-case data structures for range searching. Acta Informatica (1980) 13, 155-168.

11. Bentley J. L., Shamos M. I., A problem in multivariate statistics: algorithms, data structure and applications. Proc. 15th Allerton Conf. Commun., Contr., Comput. 1977, 193-201.

12. Bentley J. L., Stanat D. F., Analysis of range searching in quad trees. Inform. Processing Letters (1975) 3, 170-173.

13. Bolour A., Optimal retrieval algorithms for small region queries. SIAM J. Comput. (1981) 10, 721-741.

14. Chazelle B. M., Filtering search: a new approach to query-answering. Proc. 24th IEEE Annual Symp. Found. Computer Sci. 1983, 122-132.

15. Fredman M. L., A lower bound of the complexity of ortogonal range queries. J. ACM. (1981) 28, 696-705.

16. Gabow H. N., Bentley J. L., Tarjan R. E., Scaling and related techniques for geometry problems. Proc. 16th ACM Annual Symp. Theory Comput. 1984, 135-143.

17. Lee D. T., Wong C. K., Worst case analysis for region and partial region searches in multidimensional binary search trees and balansed quad trees. Acta Informatica (1977) 9, 23-29.

18. Lee D. T., Wong C. K., Quintari trees: a file structures for multidimensional database system. ACM Trans. Database Syst. (1980) 1, №1, 339-353.

19. Lueker G. S., A data structure for ortogonal range queries. Proc. 19th Annual IEEE Symp. Found. Computer Sci. (1978), 28-34.

20. Lueker G. S., Willard D. E., A data structure for dynamic range queries. Inform. Processing Letters (1982) 15, №5, 209-213.

21. Saxe J. B., On the number of range queries in k-space. Discr. Appl. Math. (1979) 1, 217-225.

22. Willard D. E. Predicate-oriented database search algorithms. Ph. D. Dissertation, Harvard Univ., Cambridge, MA, 1978. 
23. Gasanov E. E., Kuznetsova I. V., On one method to decrease average search time. Abstr. First Turkish World Math. Symp. Elazig, Turkey, 1999, 135.

24. Абдул Саттар А. В., Функциональная мера сложности вычислений в автоматных схемах. Автореферат диссер. канд. физико-матем. наук. МГУ, Москва, 1994.

25. Гасанов Э. Э., Кузнецова И. В., Оценки функциональной сложности двумерной задачи интервального поиска. Тезисы докл. ХІІ Международной конб. "Пробл. теоретич. киберн.", Нижний Новгород, 1999, 47.

26. Гасанов Э. Э., Информационно-графовая модель хранения и поиска данных. Интеллехт. системи (1998) 3, №3-4, 163-192.

27. Гасанов Э. Э., Фунхчионально-сетевые базы данных и сверхбыстрые алгоритмы поиска. Изд. центр РГГУ, Москва, 1997.

Статья поступила 18.01.2001. 